Linköping Studies in Science and Technology.

Dissertations, No. 1880

\title{
High order summation-by-parts based approximations for discontinuous and nonlinear problems
}

\author{
Cristina La Cognata
}

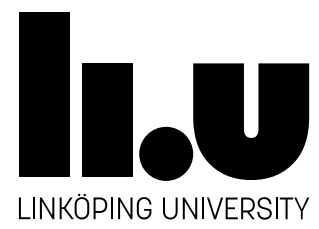

Department of Mathematics, Division of Computational Mathematics Linköping University, SE-581 83 Linköping, Sweden

Linköping 2017 
Linköping Studies in Science and Technology. Dissertations, No. 1880

High order summation-by-parts based approximations for discontinuous and nonlinear problems

Copyright (c) Cristina La Cognata, 2017

Division of Computational Mathematics

Department of Mathematics

Linköping University

SE-581 83, Linköping, Sweden

cristina.la.cognata@liu.se

www.liu.se/mai/ms

Typeset by the author in $\mathrm{LAT}_{\mathrm{E}} \mathrm{X} 2 \mathrm{e}$ documentation system.

ISSN 0345-7524

ISBN 978-91-7685-452-5

Printed by LiU-Tryck, Linköping, Sweden 2016 
To my grandmother.

She knew what my way would be before anybody else. 

There are no solved problems; there are only problems that are more or less solved.

\section{JULES HENRI POINCARÉ}





\section{Abstract}

Numerical approximations using high order finite differences on summation-byparts (SBP) form are investigated for discontinuous and fully nonlinear systems of partial differential equations. Stability and conservation properties of the approximations are obtained through a weak imposition of interface and boundary conditions with the simultaneous-approximation-term (SAT) technique. The SBP-SAT approximations replicate the continuous integration by parts rule. From this property, well-posedness and integral properties of the continuous problem are mimicked, and energy estimates leading to stability are obtained.

The first part of the thesis focuses on the simulations of discontinuous linear advection problems. An artificial interface is introduced, separating parts of the spatial domain characterized by different wave speeds. A set of flexible stability conditions at the interface are derived, which can be adapted to yield conservative or non-conservative approximations. This model can be interpreted as a simplified version of nonlinear problems involving jumps at shocks, or as a prototypical of wave propagation through different materials.

In the second part of the thesis, the vorticity/stream function formulation of the nonlinear momentum equation for an incompressible inviscid fluid is considered. SBP operators are used to derive a new Arakawa-like Jacobian with mimetic properties by combining different consistent approximations of the convection terms. Energy and enstrophy conservation is obtained for periodic problems using schemes with arbitrarily high order of accuracy. These properties are crucial for long-term numerical calculations in climate and weather forecasts or ocean circulation predictions.

The third and final contribution of the thesis is dedicated to the incompressible Navier-Stokes problem. First, different completely general formulations of energy bounding boundary conditions are derived for the nonlinear equations. The boundary conditions can be used at both far field and solid wall boundaries. The discretisation in time and space with weakly imposed initial and boundary conditions using the SBP-SAT framework is proved to be stable and the divergence free condition is approximated with the design order of the scheme. Next, the same formulations are considered in a linearised setting, whereupon the spectra associated with the initial boundary value problem and its SBP-SAT discretisation are derived using the Laplace-Fourier technique. The influence of different boundary conditions on the spectrum and in particular the convergence to steady state is studied. 



\section{Sammanfattning på svenska}

Numeriska approximationer av ekvationer som styr fysikaliska lagar är avgörande i många tillämpningar. Förutom en matematisk modell som kan fånga huvuddragen i ett verkligt problem är det nödvändigt att kunna utföra tillförlitliga simuleringar.

Denna avhandling behandlar numeriska approximationer som med hög noggrannhet bevarar både rent matematiska aspekter av ekvationerna så väl som viktiga egenskaper hos modellen. Dessutom ges särskild uppmärksamhet åt modeller med diskontinuiteter och icke-linjära beteenden.

Den första delen av avhandlingen handlar om diskontinuerliga problem. Det fysiska rummet kan ha olika egenskaper i olika regioner, något som kan resultera i instabila lösningar. Tillvägagångssättet består av att införa artificiella gränssnitt som skiljer dessa regioner åt. På detta sätt kan varje region behandlas separat, men på liknande sätt. Exempel på naturliga tillämningsområden är vågutbredning genom olika material och jordbävningssimuleringar.

I den andra delen av avhandlingen visar vi att om den numeriska approximationen imiterar partiell integration, då följer också de väsentliga egenskaperna hos modellen på ett naturligt sätt. Att fysikaliska egenskaper bevaras är nödvändigt för att bibehålla stabilitet under långa simuleringstider för bland annat geofysiska problem.

Den sista delen av avhandlingen är ägnas åt en av de mest använda modellerna inom strömningsmekanik, nämligen Navier-Stokes ekvationer. Studien fokuserar på härledningen av randvillkor som garanterar att lösningen inte växer på ett oförutsett och okontrollerat vis. Slutligen visas att de härledda randvillkoren på ett korrekt och noggrant sätt återskapar den dissipativa mekanism som ger upphov till jämviktstillstånd. 



\section{List of Papers}

This thesis is based on the following papers, which will be referred to in the text by their roman numerals.

I. Cristina La Cognata and Jan Nordström. "Well-posedness, stability and conservation for a discontinuous interface problem." BIT Numerical Mathematics 56.2 (2016): 681-704.

II. Chiara Sorgentone, Cristina La Cognata and Jan Nordström. "A new high order energy and enstrophy conserving Arakawa-like Jacobian differential operator." Journal of Computational Physics 301 (2015): 167-177.

III. Jan Nordström and Cristina La Cognata. "Energy stable boundary conditions for the nonlinear incompressible Navier-Stokes equations." Technical report LiTH-MAT-R-2017/09-SE. Submitted (2017).

IV. Cristina La Cognata and Jan Nordström. "Spectral analysis of the incompressible Navier-Stokes equations with different boundary conditions." Technical report LiTH-MAT-R-2017/10-SE.

I wrote Paper I, derived most of the theory with the assistance and editorial support of my adviser Prof. J. Nordström and wrote all the codes used for the numerical tests, as well as the manuscript.

Together with C. Sorgentone, I developed the theory and performed the numerical experiments of Paper II. J. Nordström assisted us by providing crucial suggestions, generous feedback and editorial support.

Paper III was developed jointly with the first author. I contributed to the derivation of the theoretical results and wrote most of the manuscript.

In Paper IV, I elaborated on the theoretical results from Paper III with the aid of J. Nordström, produced all the numerical calculations and wrote the manuscript. 



\section{Acknowledgements}

This is not just the end of a thesis but also, and mostly, the conclusion of an adventure. A long journey full of peeks of very intense joy and, sometimes, moments of deep pain. These five years have changed me quite a lot and all the good new sides of me are merit of the special people that I met.

Who knows me well would say that writing this last part would be harder for me than for any other chapter of this work. Probably, they would also say that this is because I am a pretty cold, rational and distant person. Indeed, they would be fairly right. But who knows me even better would say, instead, that it is hard for me because I very rarely let myself to release the emotions and write about them. But when those things happen together, one should be prepared to a huge explosion where nobody will be spared.

First of all I want to thank my supervisor Professor Jan Nordström for his unbelievable level of commitment and dedication in our collaboration during these years. He thought me how to make order in my chaotic mind, keep calm and go on. All our discussions, especially the harder ones, leaded to fruitful developments from both scientific and human point of view. Among all the lessons, my favourite will always be "simple is better".

I would like to thank all my friendly colleges at MAI for making my work place more like home, also listening to my never-ending complainants about Linköping and boring comparisons with Rome. I must have monopolised not so few lunches and fika talking about those topics. In particular, I would like to thank Tomas, Hannes, Markus, Andrea, Fatemeh, Oskar and Fredrik in my research group, Nils-Hassan and Roghi for all the nice moments in- and outside the department. Andrea requires and deserves a special thanks among them. You have been a very good friend and I will never be glad enough for your help and having saved me when I really needed it.

During these five years I lived in Linköping and Stockholm, where I made really good friends. I would like to thank my Stockholm friends Nima, Enrico, Clio and Nicolas for hosting and feed with good food, but mostly with marvellous company. Linköping has been a difficult place to live for me but it gave me the possibility to meet really special people. First of all Kinga (my favourite Scorpio) and Emanuel, Irene with who I shared many "Thelma and Louise" kind of road trips, Fredrik, Sara and Marco (thank you for bringing super good Pizza and supplì in Linköping), Carmen and Ornella (the best fika mate ever). A special thanks to Nancy for being so sweet, caring and cooking so well. Thanks also to Chiara, with who I shared all my advisers and my first good paper. 
I want to thank my family which has contributed enormously to not make me feel far from home. Especially, my mum that has took a flight as soon as she felt I was not eating enough and in the need of care. Thanks also to Incitti, Sambusetti e Marietti which, in a way or in another, I consider part of my Roman family. Thanks to Mati for all our fantastic trips and lasting friendship.

Now is the time for the big names of this adventure.

Thanks to Luca-Biancofiore (because you have to say it together) and Onofrio (marchese del Grillo) for making my life so Nanni-Morettiana, Sorrentiniana and a little Fantozziana.

Ota, my dear J, I put you among the people that contributed to my Swedish experience because you were never out of it. Our friendship tastes more about love than anything else; you complete me and give me the certainty that "all of this" has a meaning, that we still have to caught. But, we will. I hope.

A., thank you for talking directly to my soul and making it to shine. Thanks for giving to the numbers from 1 (my favourite) to 45-ish (we never have really counted them) such an exciting meaning. Sooner or later, we will reply to the question "Who knows what does it mean?". Maybe, we know already. I will wait.

Viktor, "you are" this experience. You made me to fall in love of Maths day by day, improved my work, papers, thesis, MATLAB codes, English like nobody else and introduced me to the wonderful Skänska dialect. But, most of all, you have been the first great Love and Pain of my life ("Odi et amo." Catullo said two thousand years ago) and, no matter what or who, you will be in my heart anyway, everywhere, always...

Nicolò, I made you wait. I decided to thank you for last because this long adventure started with our fated encounter and you know how much I like cycles. You are the best friend that a person can imagine. You know me so well and I adore when there is absolutely no need to explain anything to each other. But, we do it anyway because we love speculations and investigations. Not surprisingly, we are researchers. I want you to know that there will always be a free seat close to me for you. 


\section{Contents}

Abstract $\quad$ V

Sammanfattning på svenska vii

List of Papers ix

Acknowledgements $\quad$ xi

1 Introduction 1

2 Well-posedness and stability 3

3 The continuous problem $\mathbf{5}$

3.1 A model problem ................. 5

3.2 Weak boundary conditions . . . . . . . . . . . . 6

3.3 The nonlinear case . . . . . . . . . . . . . . 6

4 The discrete problem 9

4.1 SBP operators . . . . . . . . . . . . . . . 9

4.1.1 The first derivative SBP operator . . . . . . . . . . . . 9

4.1.2 The second derivative SBP operator . . . . . . . . . . 10

4.1.3 Multi-dimensional SBP approximations . . . . . . . . . 11

4.2 The SAT procedure . . . . . . . . . . . . . . 11

4.2.1 The semi-discrete single domain problem . . . . . . . 11

4.2.2 The semi-discrete multi-domain problem . . . . . . . . . 12

4.2.3 The fully discrete single domain problem . . . . . . . . 13

5 Discontinuous interface problems $\mathbf{1 5}$

5.1 Stability and conservation . . . . . . . . . . . . . 17

5.2 Spectrum analysis and strict stability . . . . . . . . . . . 17

6 Mimetic SBP properties $\quad 21$

6.1 The vorticity equation . . . . . . . . . . . . . . . 21

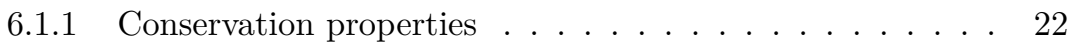

6.1.2 Operator splitting ................. . . . . . . . . . . . . . 22

6.2 Jacobian operators on SBP form . . . . . . . . . . . . . 22

6.2.1 The order of accuracy . . . . . . . . . . . . 23 
7 Boundary conditions for the Navier-Stokes equations $\quad 25$

7.1 The continuous problem . . . . . . . . . . . . 25

7.1.1 The energy method and the boundary conditions . . . . 26

7.2 The discrete problem . . . . . . . . . . . . . . . . . . 28

7.2.1 The semi-discrete formulation . . . . . . . . . . 28

7.2.2 The fully-discrete formulation . . . . . . . . . . . . . 29

7.3 Spectral analysis . . . . . . . . . . . . . . . . . 30

7.3.1 The continuous and discrete spectrum . . . . . . . . 30

7.3.2 Predicted and practical convergence rates . . . . . . . 31

7.4 Comparison of different boundary conditions . . . . . . . . . . 31

8 Summary of papers $\quad 35$

$\begin{array}{ll}\text { References } & \mathbf{3 7}\end{array}$

I. Well-posedness, stability and conservation for a discontinuous interface problem ........................... 39

II. A new high order energy and enstrophy conserving Arakawa-like Jacobian differential operator . . . . . . . . . . . . . . 65

III. Energy stable boundary conditions for the nonlinear incompressible Navier-Stokes equations . . . . . . . . . . . . . . 79

IV. Spectral analysis of the incompressible Navier-Stokes equations with different boundary conditions . . . . . . . . . . . . 103 


\section{Introduction}

Natural phenomena arise from interactions among physical processes. Mathematically, these processes are modelled using differential equations. The equations are often nonlinear, such as in the modelling of wave fronts in the ocean, or the formation of hurricanes and storms. Frequently, they also encompass discontinuities, e.g. when light passes across a material interface, or when an earthquake sends vibrations through the ground, water and air.

It is in general non-trivial to determine whether the resulting equations admit a well-behaved solution. This is nonetheless necessary in order to numerically obtain an accurate and reliable approximation of the solution. This thesis is dedicated to the study of discontinuous and nonlinear problems, and their approximations using high order numerical methods.

The first part of the thesis deals with advection problems with discontinuous coefficients and solutions. The questions of well-posedness, stability and conservation are studied in a general setting and addressed by deriving continuous and discrete conditions. In the second part, it is shown how to preserve analytical properties of differential operators in the numerical approximations. In particular, nonlinear products of convection terms can be reformulated in order to conserve important quantities. The last contribution of this thesis consists of a study of energy stable boundary conditions for the incompressible NavierStokes problem in both linear and nonlinear form. Two flexible formulations are derived, that can be adapted to different types of conditions. Finally, the influence of different boundary conditions on the convergence to steady state is studied for both the continuous and discrete problem.

Throughout the thesis, differential equations will be discretised using high order finite difference methods on summation-by-parts (SBP) form $[19,16,8]$. By combining the SBP discretisation with weakly imposed initial, boundary and interface conditions using the simultaneous-approximation-term (SAT) procedure $[3,17]$, the resulting SBP-SAT schemes become stable and high order accurate.

The thesis is organised as follows: In Chapter 2, the concepts of well-posed problems and the stability of numerical approximations are presented. In Chapter 3 , the energy method as a fundamental tool in all the analysis of this work is introduced. Chapter 4 contains an introduction to the SBP-SAT framework and a few of its applications. Chapter 5,6 and 7 are dedicated to the new 
contributions from this work. Although the discussions are carried out for high order finite difference methods, most of the theoretical findings and numerical results are equally valid for other approximations on SBP-SAT form, such as finite volume, finite elements, spectral elements, discontinuous Galerkin and flux reconstruction schemes. 


\section{Well-posedness and stability}

Well-posedness and stability are two fundamental concepts in numerical approximations of differential equations. To introduce these concepts, consider a general initial-boundary-value-problem (IBVP) on the spatial domain $\Omega$ with boundary $\partial \Omega$

$$
\begin{aligned}
u_{t}+P u & =F, & x \in \Omega, t & \geq 0, \\
H u & =g, & x \in \partial \Omega, t & \geq 0, \\
u & =f, & x \in \Omega, t & =0 .
\end{aligned}
$$

Here, $P$ is a spatial differential operator, $H$ is a boundary operator and $F, g$ and $f$ are given forcing, boundary and initial data, respectively. The data are assumed to be smooth and compatible functions. The IBVP (2.1) is well-posed if a unique solution exists and there is an estimate in terms of data of the form

$$
\|u\|_{I}^{2} \leq K\left(\|f\|_{I I}^{2}+\|F\|_{I I I}^{2}+\|g\|_{I V}^{2}\right) .
$$

in $(2.2), K$ is bounded and may depend on the time, but not on the data. The norms involved are in general different.

An important implication of a well-posed problem with an estimate of the form (2.2) is that the solution depends continuously on the data. Therefore, small perturbations in the data cause small perturbations in the solution. This can easily be seen in the case of linear operators $P$ and $H$ by considering the solution $v$ of the perturbed problem with data $F+\delta F, g+\delta g$ and $f+\delta f$. Let $w=v-u$ be the perturbation of the solution satisfying the IBVP

$$
\begin{aligned}
w_{t}+P w & =\delta F, & x \in \Omega, t & \geq 0, \\
H w & =\delta g, & x \in \partial \Omega, t & \geq 0, \\
w & =\delta f, & x \in \Omega, t & =0 .
\end{aligned}
$$

For this problem, the estimate (2.2) becomes

$$
\|w\|_{I}^{2} \leq K\left(\|\delta f\|_{I I}^{2}+\|\delta F\|_{I I I}^{2}+\|\delta g\|_{I V}^{2}\right)
$$

Hence, small perturbations in the data lead to small perturbations of the solution. 
Stability is the discrete counterpart of well-posedness. Roughly speaking, the approximations of an IBVP is stable if a discrete estimate in terms of data, similar to (2.2), can be derived. Also in the discrete case, this implies that small perturbations in the data lead to small and controlled variations in the numerical solution.

Since well-posedness and stability are intimately related, every investigation in this thesis will contain the study of well-posedness of a continuous model problem followed by the stability analysis of the discretised version. 


\section{The continuous problem}

For many models in applied sciences and engineering, the question of wellposedness can be addressed by applying the so called energy method. This is the case for the linear IBVP (2.1). More precisely, if the energy method applied to (2.1) yields an energy estimate of the form (2.2), uniqueness follows directly and existence can be guaranteed by imposing the correct (minimal) number of boundary conditions. We will illustrate the procedure in a simplified setting below.

\subsection{A model problem}

Consider the IBVP for a scalar advection problem in one space dimension

$$
\begin{array}{rlrl}
u_{t}+u_{x} & =F, & 0 \leq x \leq 1, t \geq 0, \\
u & =g(t), & x & =0, t \geq 0, \\
u & =f(x), & 0 \leq x \leq 1, t=0 .
\end{array}
$$

Consider also the inner product and the induced norm

$$
(u, v)=\int_{0}^{1} u(x) v(x) d x, \quad\|u\|^{2}=(u, u) .
$$

The energy method applied to (3.1) (multiplying the equation by the solution, integrating by parts and imposing the boundary conditions) yields

$$
\frac{d}{d t}\|u(\cdot, t)\|^{2}=g(t)^{2}-u(1, t)^{2}+2 \int_{0}^{1} u(\cdot, t) F(\cdot, t) d x .
$$

By using the inequality $2 \int_{0}^{1} u F d x \leq \eta\|u\|^{2}+\|F\|^{2} / \eta$ (with a positive constant $\eta),(3.2)$ becomes

$$
\frac{d}{d t}\|u(\cdot, t)\|^{2}-\eta\|u(\cdot, t)\|^{2} \leq g(t)^{2}+\|F(\cdot, t)\|^{2} / \eta .
$$

Finally, by solving for $\|u\|^{2}$ at the final time $T$, one gets the estimate

$$
\|u(\cdot, T)\|^{2} \leq K e^{\eta T}\left(\|f(\cdot)\|^{2}+\int_{0}^{T}\|F(\cdot, \tau)\|^{2}+g(\tau)^{2} d \tau\right),
$$


which is of the form (2.2).

To summarise, the energy method produces an evolution equation for the norm of the solution involving boundary terms. Once the boundary conditions have been imposed and the remaining boundary terms have a clear correct sign, an energy estimate can be obtained. The energy method applied to more advanced problems is more complicated, but the core of the procedure remains the same.

\subsection{Weak boundary conditions}

The same result as the one above can be obtained by weakly imposing the boundary conditions $(3.1 \mathrm{~b})$ to $(3.1 \mathrm{a})$ as follows

$$
\begin{aligned}
u_{t}+u_{x} & =L(g-u)+F, & & 0 \leq x \leq 1, t \geq 0 . \\
u & =f(x), & & 0 \leq x \leq 1, t=0 .
\end{aligned}
$$

Here, $L$ is a lifting operator [2] defined such that, for smooth functions $\phi$ and $\psi$, it satisfies

$$
\int_{0}^{1} \phi L(\psi) d x=\left.\phi \psi\right|_{x=0}
$$

By applying the energy method to (3.4), we get

$$
\frac{d}{d t}\|u(\cdot, t)\|^{2}=g^{2}(t)-u(1, t)^{2}-(u(0, t)-g)^{2}+2 \int_{0}^{1} u(\cdot, t) F(\cdot, t) d x .
$$

By ignoring the terms with the correct sign and integrating (3.5) in time, the same energy estimate as in (3.3) is obtained.

Note that (3.5) is the same as (3.2) with an additional term coming from the energy method applied to the lifting operator. This term is zero in the continuous case since the boundary condition is identically satisfied. In the discrete setting, it is not and the term (as we will see), will provide numerical damping.

\subsection{The nonlinear case}

Well-posedness of the nonlinear version of IBVP (2.1) is still an open question and a deep discussion on this matter is beyond the scope of this thesis. Here, we briefly discuss how to relate the question of well-posedness for IBVPs with quasilinear PDEs (2.1a) to well-posedness of linearised and frozen coefficient problems.

The operator $P$ in (2.1a) is a quasilinear differential operator of order $m$ if it has the general form

$$
P(x, t, u, \partial / \partial x)=\sum_{|\nu| \leq m} A_{\nu}(x, t, u) \frac{\partial^{|\nu|}}{\partial^{\nu_{1}} x_{1} \ldots \partial^{\nu_{s}} x_{s}},
$$

where $|\nu|=\nu_{1}+\ldots+\nu_{m}$ for a multi-index $\nu=\left(\nu_{1}, \ldots, \nu_{s}\right)$ and the coefficients $A_{\nu}$ are given smooth functions. 
The following linearisation principle holds: An IBVP (2.1) with quasilinear differential operator (3.6) is well-posed at $u$ if the linear problem obtained by linearising all functions $A_{\nu}$ near $u$ is well-posed.

By freezing the coefficients $A_{\nu}$ at an arbitrary fixed point and time, the following localisation principle holds: If all frozen-coefficient problems are well-posed, then the original variable coefficient problem is well-posed.

For more details regarding well-posed IBVPs and the linearisation and localisation principles, see $[6]$.

It should be noted that the localisation principle does not hold for all variablecoefficient problem. However, without discussing this further, the differential operators of the vorticity equation in Paper II and the incompressible NavierStokes equations in Paper III belong to the classes of quasilinear operators for which this principle can be applied. 



\section{The discrete problem}

In this chapter, the SBP-SAT approximation in finite difference form for computing the solution for IBVPs is introduced. The SBP operators are high order discrete differential operators constructed to mimic the integration-by-part rule in the discrete setting. The discrete version of the energy method is applied to the discretised equations and the same procedure as in the continuous case is replicated in order to derive an estimate of the discrete solution. The combination of the SBP technique with the SAT procedure for imposing initial, boundary and interface conditions weakly, yield SBP-SAT approximations which are high order accurate and provably stable.

\subsection{SBP operators}

We start by introducing the discrete SBP operators in the one-dimensional case. Consider a uniform mesh of $N+1$ grid points on the interval $x \in[0,1]$ with coordinates $x_{i}=i h, 0 \leq i \leq N$, where $h=1 / N$ is the spatial step. The discrete approximation of a variable $v=v(x)$ is indicated by the grid function $\mathbf{v}=\left(v_{1}, \ldots, v_{N}\right)^{T}$, where $v_{i} \approx v\left(x_{i}\right)$.

\subsubsection{The first derivative SBP operator}

The SBP approximation of the first derivative of a smooth function $v$ is given by

$$
D_{x} \mathbf{v}=P_{x}^{-1} Q_{x} \mathbf{v} \approx v_{x} .
$$

In (4.1), $P_{x}$ is a diagonal, positive definite matrix such that it forms a discrete $L^{2}$-norm, namely $\|\mathbf{v}\|_{P_{x}}^{2}=\mathbf{v}^{T} P_{x} \mathbf{v} \approx \int v^{2} d x$, associated with the discrete inner product $(\mathbf{v}, \mathbf{w})_{P_{x}}=\mathbf{v}^{T} P_{x} \mathbf{w}$. The operator $Q_{x}$ is an almost skew-symmetric matrix satisfying the SBP property

$$
Q_{x}+Q_{x}=B=-E_{0}+E_{N}
$$

where $E_{0}=\operatorname{diag}(1,0, \ldots, 0)$ and $E_{N}=\operatorname{diag}(0, \ldots, 0,1)$. By using this property, the integration-by-parts rule

$$
\int_{0}^{1} v_{x} w d x=-\int_{0}^{1} v w_{x} d x+v(1) w(1)-v(0) w(0)
$$


is mimicked by the following summation-by-parts rule

$$
\left(D_{x} \mathbf{v}, \mathbf{w}\right)_{P_{x}}=-\left(\mathbf{v}, D_{x} \mathbf{w}\right)_{P_{x}}+v_{N} w_{N}-v_{0} w_{0} .
$$

A first order derivative SBP operator $D$ is $p$-th order accurate if it approximates derivatives of polynomials up to order $p$ exactly, i.e.,

$$
D \mathbf{x}^{j}=j \mathbf{x}^{j-1}, \quad j=0, \ldots, p .
$$

Here, $\mathbf{x}=\left(x_{0}, \ldots, x_{N}\right)^{T}$ is the grid vector and the exponentiation is interpreted element-wise. In this thesis, we consider first order derivative SBP operators of order $2 p$, where $p \in\{1,2,3,4\}$ in the interior and boundary closures of order $p$. In combination with diagonal matrices $P$, the global order of accuracy is $p+1$ for pointwise stable approximations involving first order derivatives [18]. We refer to a specific SBP operator with the acronym followed by the interior and then the boundary closure order of accuracy. As an example, the second order accurate SBP21 operator $D=P^{-1} Q$ is given by the matrices

$$
Q=\left[\begin{array}{ccccc}
-\frac{1}{2} & \frac{1}{2} & & & \\
-\frac{1}{2} & 0 & \frac{1}{2} & & \\
& \ddots & \ddots & \ddots & \\
& & -\frac{1}{2} & 0 & \frac{1}{2} \\
& & & -\frac{1}{2} & \frac{1}{2}
\end{array}\right], \quad P=h\left[\begin{array}{ccccc}
\frac{1}{2} & & & & \\
& 1 & & & \\
& & \ddots & & \\
& & & 1 & \\
& & & \frac{1}{2}
\end{array}\right],
$$

where $h$ is the grid spacing.

It should be noted that the use of a diagonal $P$ is not a necessary for the construction of an accurate SBP operator. However, for problems involving variable coefficients or nonlinear problems and a non-diagonal $P$, stability cannot be proved. For more details on first order derivative operators, see [16].

\subsubsection{The second derivative SBP operator}

The SBP approximation of the second derivative can be obtained by simply applying the first derivative operator $D_{x}$ in (4.1) twice, i.e.

$$
D_{x x} \mathbf{v}=D_{x}^{2} \mathbf{v} \approx v_{x x} .
$$

Higher order discrete derivatives can be constructed similarly.

By applying the first derivative operator twice, a wide operator is obtained. Compact second order derivatives are formally defined as follows

$$
D_{x x} \mathbf{v}=P^{-1}(-M+B S) \mathbf{v} \approx v_{x x},
$$

where $M+M^{T}$ is a positive semi-definite matrix, $S$ is a first derivative operator at the boundary and $B$ is given in (4.2). When (4.4) is used for the wave equation, $M$ must be also symmetric. For more details on second derivative operators, see [8]. 


\subsubsection{Multi-dimensional SBP approximations}

Multi-dimensional approximations of partial derivatives are constructed by extending the one-dimensional operators using tensor (Kronecker) products.

Consider a two-dimensional grid of $N \times M$ points with coordinates $\left(x_{i}, y_{j}\right)$ and the discrete approximation of a variable $v=v(x, y)$ given by the vector $\mathbf{v}=\left(v_{11}, \ldots, v_{1 M}, v_{21} \ldots, v_{2 M}, \ldots, v_{N 1}, \ldots, v_{N M}\right)^{T}$, where $v_{i j} \approx v\left(x_{i}, y_{j}\right)$.

The SBP approximation of the partial derivatives of $v$ are given by

$$
D_{x} \mathbf{v}=\left(P_{x}^{-1} Q_{x} \otimes I_{M}\right) \mathbf{v} \approx \frac{\partial \mathbf{v}}{\partial x} \quad \text { and } \quad D_{y} \mathbf{v}=\left(I_{N} \otimes P_{y}^{-1} Q_{y}\right) \mathbf{v} \approx \frac{\partial \mathbf{v}}{\partial y},
$$

where $I_{d}$ is the identity matrix of dimension $d$ and $\otimes$ denotes the Kronecker product. In this case, the diagonal matrix $\left(P_{x} \otimes P_{y}\right)$ defines a two-dimensional discrete $L^{2}$-norm, such that $\|\mathbf{v}\|_{\left(P_{x} \otimes P_{y}\right)}^{2}=\mathbf{v}^{T}\left(P_{x} \otimes P_{y}\right) \mathbf{v} \approx \int v^{2} d x d y$.

The same principle can be applied to three dimensional problems and discretisation in time. For details regarding SBP approximations in time, see [12, 7].

\subsection{The SAT procedure}

The SBP finite difference approximation requires a suitable boundary treatment in order to be stable. The SAT technique, originally used for imposing boundary conditions and extended to interface and initial conditions, is the preferred choice. In this technique, the conditions are weakly imposed by adding a penalty term to the equations. There exist other ways to enforce boundary conditions like, for instance, the injection and projection methods. In the first case many situations lead to instabilities, see [5]. In the second case, the problem is reformulated and projection operators are constructed such that stability can be guaranteed [13]. However, the projection method is rather complicated.

\subsubsection{The semi-discrete single domain problem}

The semi-discrete SBP-SAT approximation of (3.1) or (3.4) is given by

$$
\mathbf{u}_{t}+D_{x} \mathbf{u}=\sigma P^{-1}\left(u_{1}-g\right) e_{0}+\mathbf{F},
$$

where $\mathbf{u}$ is the discrete variable, $\mathbf{F}$ the discrete forcing function and $e_{0}=$ $(1,0, \ldots, 0)$. In $(4.5), \sigma$ is a penalty coefficient which will be chosen to ensure stability.

By applying the discrete energy method to (4.5) (multiplying the equation from the left by $\mathbf{u}^{T} P_{x}$ and adding its transpose) and using the SBP property (4.2), we get

$$
\frac{d}{d t}\|\mathbf{u}\|_{P_{x}}^{2}=u_{0}^{2}-u_{N}^{2}+2 \sigma u_{0}^{2}-2 \sigma u_{0} g+2 \mathbf{u}^{T} P \mathbf{F}
$$


To mimic the continuous case, we choose $\sigma=-1$ and complete the square. This leads to

$$
\frac{d}{d t}\|\mathbf{u}\|_{P_{x}}^{2}=g^{2}-u_{N}^{2}-\left(u_{0}-g\right)^{2}+2 \mathbf{u}^{T} P \mathbf{F},
$$

which is the discrete version of (3.5). Finally, by using the discrete inequality $2 \mathbf{u}^{T} P \mathbf{F} \leq \eta\|\mathbf{u}\|_{P_{x}}^{2}+\|\mathbf{F}\|_{P_{x}}^{2} / \eta$ (with a positive constant $\eta$ ) and integrating in time, one gets the discrete energy estimate

$$
\|\mathbf{u}\|_{P_{x}}^{2} \leq K e^{\eta t}\left(\|\mathbf{f}\|_{P_{x}}^{2}+\int_{0}^{t}\|\mathbf{F}\|_{P_{x}}^{2}+g(\tau)^{2} d \tau\right)
$$

where $\mathbf{f}$ is the discrete initial condition. This estimate is similar to (3.3) and implies that the SBP-SAT approximation is stable.

\subsubsection{The semi-discrete multi-domain problem}

Problems that require geometrical flexibility can be treated in the SBP-SAT framework by employing stable multi-block approximations. This strategy allows for different mesh sizes and orders of accuracy in different regions.

To exemplify the technique, consider two advection equations in two different domains

$$
\begin{aligned}
u_{t}+u_{x}=0, & x \leq 0, t \geq 0, \\
v_{t}+v_{x}=0, & x \geq 0, t \geq 0,
\end{aligned}
$$

connected at $x=0$ by the interface condition $v(0, t)=u(0, t)$.

By ignoring the boundary conditions at the outer boundaries, we can write the approximation of (4.6) with weakly imposed interface conditions as

$$
\begin{aligned}
\mathbf{u}_{t}+P_{l}^{-1} Q_{l} \mathbf{u} & =P_{l}^{-1} \sigma_{L}\left(u_{N}-v_{0}\right) e_{N} \\
\mathbf{v}_{t}+P_{r}^{-1} Q_{r} \mathbf{v} & =P_{r}^{-1} \sigma_{R}\left(v_{0}-u_{N}\right) e_{0} .
\end{aligned}
$$

Here, $e_{N}=(0, \ldots, 0,1)$ and $e_{0}=(1,0 \ldots, 0)$ have length of the left and right mesh, respectively, $u_{N}$ is the last element of the grid function $\mathbf{u}$ and $v_{0}$ is the first element of v. $P_{l, r}^{-1} Q_{l, r}$ are SBP operators, possibly different, operating on the left and right domain, respectively.

To apply the semi-discrete energy method, we multiply (4.7) from the left with $u^{T} P_{l}$ and (4.8) with $v^{T} P_{r}$. By summing the results, we get

$$
\frac{d}{d t}\left[\|\mathbf{u}\|_{P_{l}}^{2}+\|\mathbf{v}\|_{P_{r}}^{2}\right]=\mathrm{IT},
$$

where IT is a quadratic form given by

$$
\mathrm{IT}=\left(\begin{array}{c}
u_{N} \\
v_{0}
\end{array}\right)^{T} H\left(\begin{array}{c}
u_{N} \\
v_{0}
\end{array}\right), \quad H=\left[\begin{array}{cc}
\left(-1+2 \sigma_{L}\right) & -\left(\sigma_{L}+\sigma_{R}\right) \\
-\left(\sigma_{L}+\sigma_{R}\right) & \left(1+2 \sigma_{R}\right)
\end{array}\right]
$$


Hence, we have an energy estimate if $\mathrm{IT} \leq 0$. This requires $\mathrm{H}$ to be a negative semi-definite matrix, which can be obtained by choosing $\sigma_{L}=\sigma_{R}+1$ and $\sigma_{R} \leq-1 / 2$.

This multi-block technique was extensively studied in paper I, where the discontinuities in the model problem were treated as interface conditions.

\subsubsection{The fully discrete single domain problem}

Consider a computational domain with $N$ grid points on the spatial interval and $L$ time levels. By recalling the notation introduced in Section 4.1.3, the fully-discrete approximation of a variable $u=u(t, x)$ is a vector of length $L N$ arranged as

$$
\mathbf{u}=\left[\begin{array}{c}
\vdots \\
{[\mathbf{u}]_{k}} \\
\vdots
\end{array}\right],[\mathbf{u}]_{k}=\left[\begin{array}{c}
\vdots \\
u_{k i} \\
\vdots
\end{array}\right], \quad \text { where } u_{k i} \approx u\left(t_{k}, x_{i}\right)
$$

The fully discrete approximation of (3.4) with zero forcing function and weakly imposed boundary and initial conditions is

$$
\begin{aligned}
\left(D_{t} \otimes I_{N}\right) \mathbf{u}+ & \left(I_{L} \otimes D_{x}\right) \mathbf{u}= \\
& \sigma_{x}\left(I_{L} \otimes P_{x}^{-1} E_{0 x}(\mathbf{u}-\mathbf{g})\right)+\sigma_{t}\left(P_{t}^{-1} E_{0 t}(\mathbf{u}-\mathbf{f}) \otimes I_{N}\right),
\end{aligned}
$$

where $E_{0 x}$ and $E_{0 t}$ have the same dimension as the corresponding SPB operators. Here, the grid functions $\mathbf{g}$ and $\mathbf{f}$ contain the boundary and initial data, respectively, at the proper positions.

We apply the discrete energy method to (4.9) (multiplying the equation from the left by $\mathbf{u}^{T}\left(P_{t} \otimes P_{x}\right)$ and adding its transpose) and use the SBP property. By also choosing suitable penalty coefficients $\left(\sigma_{x}=\sigma_{t}=-1\right)$ and rearranging, we get

$$
\left\|\mathbf{u}_{L}\right\|_{P_{x}}^{2}=\|\mathbf{f}\|_{P_{x}}^{2}+\|\mathbf{g}\|_{P_{t}}^{2}-\left\|\left(E_{0 t} \otimes I_{x}\right)(\mathbf{u}-\mathbf{f})\right\|_{\left(P_{t} \otimes P_{x}\right)}^{2}-\left\|\left(I_{t} \otimes E_{0 x}\right)(\mathbf{u}-\mathbf{g})\right\|_{\left(P_{t} \otimes P_{x}\right)}^{2} .
$$

Note that we have obtained a fully discrete version of (3.3) with additional damping terms coming from the spatial and temporal penalty terms. 



\section{Discontinuous interface problems}

The interface treatment described in Section 4.2.2 can be extended to advection equations with different wave-speeds. In addition, one can require that the solution satisfies a jump-condition at the interface. In this general setting, fundamental properties such as well-posedness, stability and conservation were investigated in Paper I.

Consider an interface problem defined by the coupled equations

$$
\begin{aligned}
u_{t}+a u_{x} & =0, \quad x \leq 0, t \geq 0, \quad \text { with } \quad v(0, t)=c u(0, t) \\
v_{t}+b v_{x} & =0, \quad x \geq 0, t \geq 0,
\end{aligned}
$$

where the wave-speeds $a$ and $b$ are real and positive. In (5.1), $c$ is a real constant which makes the solution discontinuous at the interface $x=0$ when it is different from one.

By applying the energy method to (5.1), using a modified $L^{2}$-norm and ignoring the outer boundary terms, we get

$$
\int_{-\infty}^{0} u\left[u_{t}+a u_{x}\right] d x+\int_{0}^{\infty} \alpha_{c} v\left[v_{t}+b v_{x}\right] d x=0
$$

where $\alpha_{c}$ is a positive free weight parameter. Integration-by-parts shows that an energy estimate is obtained by choosing $\alpha_{c}$ such that

$$
-a+\alpha_{c} b c^{2} \leq 0
$$

holds. Thus, the interface problem (5.1) is well-posed for positive $a, b$ and $c \in \mathbb{R}$. The conservation conditions are derived by considering the weak formulation of (5.1) given by

$$
\int_{-\infty}^{\infty}[\phi w]_{0}^{t} d x-\int_{-\infty}^{\infty} \int_{0}^{t}\left[\phi_{t}+\phi_{x} \bar{u}\right] w d x d t+\int_{0}^{t} \phi w[a-b c]_{x=0} d t=0
$$

where

$$
w(x, t)= \begin{cases}u(x, t), & x \leq 0, t \geq 0 \\ v(x, t), & x \geq 0, t \geq 0\end{cases}
$$


In (5.2), $\phi(x, t) \in C^{\infty}$ has compact support in the spatial interval $(-\infty, \infty)$, while $\bar{u}=a$ for $x \leq 0$ and $\bar{u}=b$ for $x>0$. Hence, all the terms at the interface vanish, resulting in a conservative problem, if

$$
c=a / b \text {. }
$$

Using the notation of Section 4.2.2, the SBP-SAT approximation of (5.1) with weakly imposed interface conditions is

$$
\begin{aligned}
& \mathbf{u}_{t}+a P_{l}^{-1} Q_{l} \mathbf{u}=P_{l}^{-1} \sigma_{L}\left(c u_{N}-v_{0}\right) e_{N} \\
& \mathbf{v}_{t}+b P_{r}^{-1} Q_{r} \mathbf{v}=P_{r}^{-1} \sigma_{R}\left(v_{0}-c u_{N}\right) e_{0}
\end{aligned}
$$

with $e_{N}=(0, \ldots, 0,1)$ and $e_{0}=(1,0 \ldots, 0)$ of the proper length.

The approximation (5.4) can be written in a compact matrix form as follows

$$
\left(\begin{array}{l}
\mathbf{u} \\
\mathbf{v}
\end{array}\right)_{t}=P^{-1} \tilde{Q}\left(\begin{array}{l}
\mathbf{u} \\
\mathbf{v}
\end{array}\right)
$$

where

$$
P=\left[\begin{array}{cc}
P_{l} & 0 \\
0 & P_{r}
\end{array}\right], \tilde{Q}=-Q_{\Lambda}+\Sigma, \text { and } Q_{\Lambda}=\left[\begin{array}{cc}
a Q_{l} & 0 \\
0 & b Q_{r}
\end{array}\right] .
$$

The penalty matrix $\Sigma$, which contains the penalties coefficients, is zero everywhere except at the boundary and interface points.

The energy method applied to (5.4) (or equivalently to (5.5)) gives

$$
\frac{d}{d t}\left[\|\mathbf{u}\|_{P_{l}}^{2}+\alpha_{d}\|\mathbf{v}\|_{P_{r}}^{2}\right]=\mathrm{IT},
$$

where $\alpha_{d}$ is a positive weight (not necessarily the same as in the continuous case). The right-hand side term IT is given by

$$
\mathrm{IT}=\left(\begin{array}{c}
u_{N} \\
v_{0}
\end{array}\right)^{T} H\left(\begin{array}{c}
u_{N} \\
v_{0}
\end{array}\right), \quad H=\left[\begin{array}{cc}
\left(-a+2 c \sigma_{L}\right) & -\left(\sigma_{L}+\alpha_{d} c \sigma_{R}\right) \\
-\left(\sigma_{L}+\alpha_{d} c \sigma_{R}\right) & \alpha_{d}\left(b+2 \sigma_{R}\right)
\end{array}\right]
$$

To get an energy estimate, $\mathrm{H}$ must be negative semi-definite. This can be achieved by choosing $\sigma_{L}$ and $\sigma_{R}$ such that both the following conditions hold

$$
\begin{aligned}
\left(-a+2 c \sigma_{L}\right)+\alpha_{d}\left(b+2 \sigma_{R}\right) & \leq 0 \\
\left(-a+2 c \sigma_{L}\right) \alpha_{d}\left(b+2 \sigma_{R}\right)-\left(\sigma_{L}+\alpha_{d} c \sigma_{R}\right)^{2} & \geq 0
\end{aligned}
$$

By rewriting (5.4) in weak form, one obtains the discrete conservation condition

$$
\sigma_{R}=\sigma_{L}-b .
$$




\subsection{Stability and conservation}

In this section, we will show that conservation and stability are two independent properties of the approximation (5.4). More precisely, there exist penalty interface coefficients which lead to accurate and stable SBP-SAT schemes which can be made conservative or non-conservative.

Consider a well-posed interface problem without the conservation condition (5.3). The SBP-SAT approximation (5.4) is stable for all parameters $a, b$ and $c$ when the penalty coefficients $\sigma_{L}$ and $\sigma_{R}$ satisfy both

$$
\sigma_{R} \leq-b / 2
$$

and

$$
\frac{b+\sigma_{R}-\sqrt{\left(b+2 \sigma_{R}\right)\left(b-\theta\left(\frac{a}{c}\right)\right)}}{\theta} \leq \sigma_{L} \leq \frac{b+\sigma_{R}+\sqrt{\left(b+2 \sigma_{R}\right)\left(b-\theta\left(\frac{a}{c}\right)\right)}}{\theta} .
$$

Furthermore, the relation $\theta=1 /\left(\alpha_{d} c\right) \geq b c / a$ must hold for real penalty coefficients.

Consider now a conservative problem (i.e., (5.3) holds). A stable SBP-SAT approximation (not necessarily conservative) is obtained if the penalty parameters $\sigma_{L}$ and $\sigma_{R}$ satisfy (5.7) and

$$
\frac{b+\sigma_{R}-\sqrt{b\left(b+2 \sigma_{R}\right)(1-\theta)}}{\theta} \leq \sigma_{L} \leq \frac{b+\sigma_{R}+\sqrt{b\left(b+2 \sigma_{R}\right)(1-\theta)}}{\theta}
$$

with $\theta=b /\left(a \alpha_{d}\right) \geq 1$.

Finally, conditions (5.3) and (5.6) lead to a stable and conservative SBP-SAT approximation (5.4) if

$$
\frac{b}{1-\sqrt{\theta}} \leq \sigma_{L} \leq \frac{b}{1+\sqrt{\theta}} \quad \text { with } \quad \theta=b /\left(a \alpha_{d}\right) \geq 1
$$

In Figure 5.1 (from paper I), we show a few frames of the time-evolution of a conservative solution to (5.1). The initial condition is zero. The boundary data $g(t)=\sin (4 \pi(-1+3 t))$ is weakly imposed at the inflow boundary using the SAT procedure. The wave propagates with velocity $\mathrm{a}=2$ in the left domain and $\mathrm{b}=1$ in right domain. The jump condition is $\mathrm{c}=2$. The simulation is done using a standard fourth order accurate Runge-Kutta method in time and a fifth order accurate SBP-SAT approximation in space.

\section{$5.2 \quad$ Spectrum analysis and strict stability}

In this section, we study the influence of the interface treatment on the continuous and discrete spectrum. 

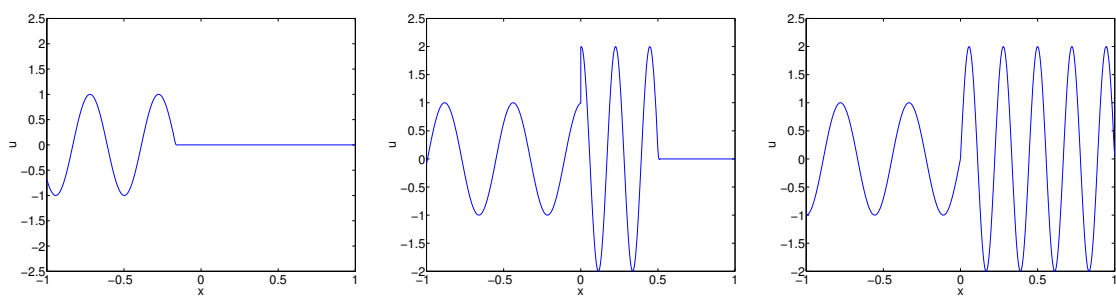

Figure 5.1: Time-evolution of a wave function satisfying a conservative jump condition and computed with a conservative approximation.

The discrete spectrum is given by the eigenvalues of $P^{-1} \tilde{Q}$ in (5.5). The continuous spectrum of (5.1) (derived using the Laplace Transform technique) is given by the infinite sequence

$$
s=\frac{a b}{a+b}[\log (|c d|)+2 i \pi k], \quad k \in \mathbf{Z} \quad \text { with } \quad c d \neq 0 .
$$

The real constant $d$ defines the outer boundary closure through $u(-1, t)-$ $d v(1, t)=0$. In Figure 5.2 (from Paper I), the discrete spectrum and the continuous one for a conservative problem and conservative approximation are presented. As can be seen, the spectra consist only of eigenvalues with negative real parts. This implies well-posedness and a stable semi-discretisation.

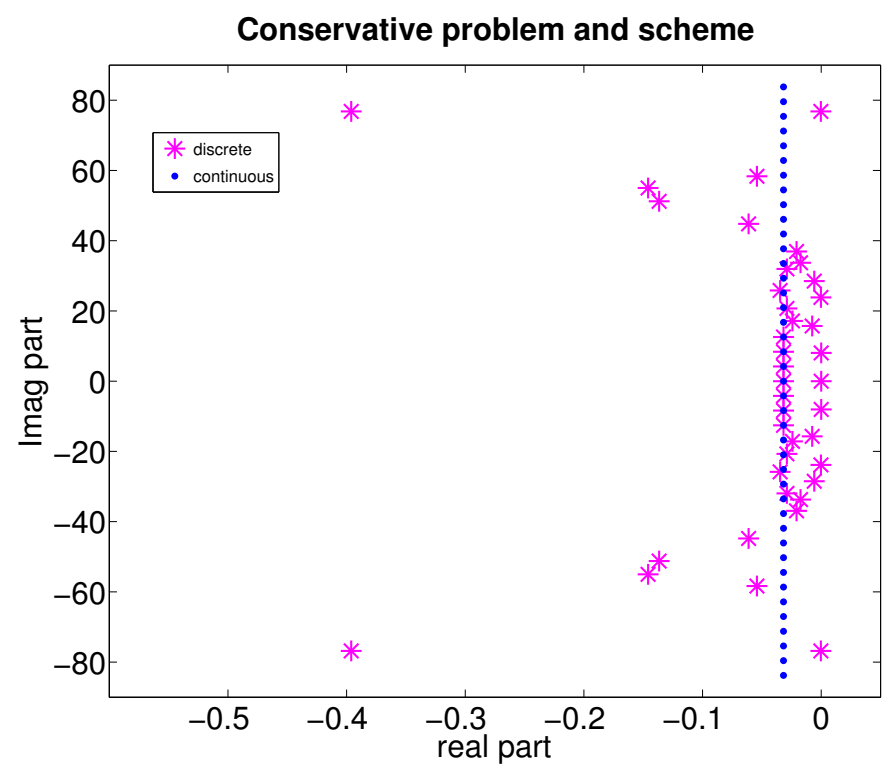

Figure 5.2: Continuous and semi-discrete 4 th order accurate spectrum. 
However, Figure 5.2 also shows that some of the discrete eigenvalues lie to the right of the continuous spectrum. The time growth rate of a strictly stable approximation is bounded by the growth rate of the corresponding continuous problem [10]. Hence, having the eigenvalues of the discrete spectrum on the left side of the continuous spectrum is preferable. By adding a suitable artificial dissipation operator to the approximation (5.4), one can shift the discrete spectrum to the left of the continuous one without reducing the accuracy [9]. Figures 5.3a-5.3b (from Paper I) show a close-up of the spectrum of a conservative approximation with and without artificial dissipation. With added dissipation, the discrete spectrum converges from the left implying strict stability. 


\section{Convergence of conservative scheme without dissipation}

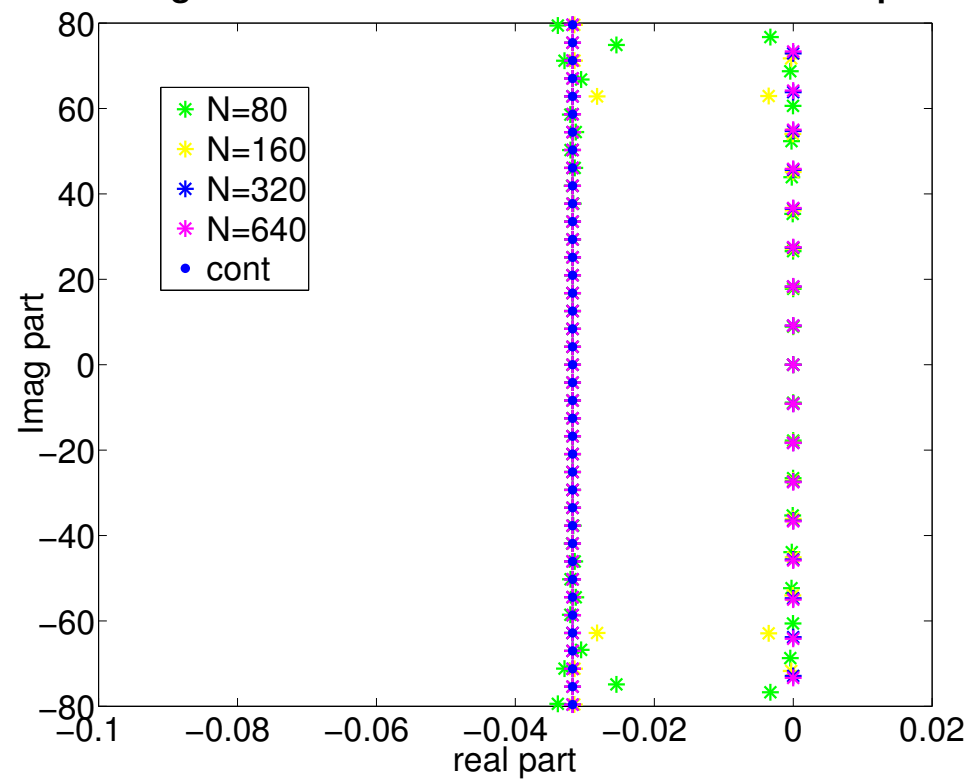

(a)

Convergence of conservative scheme with dissipation

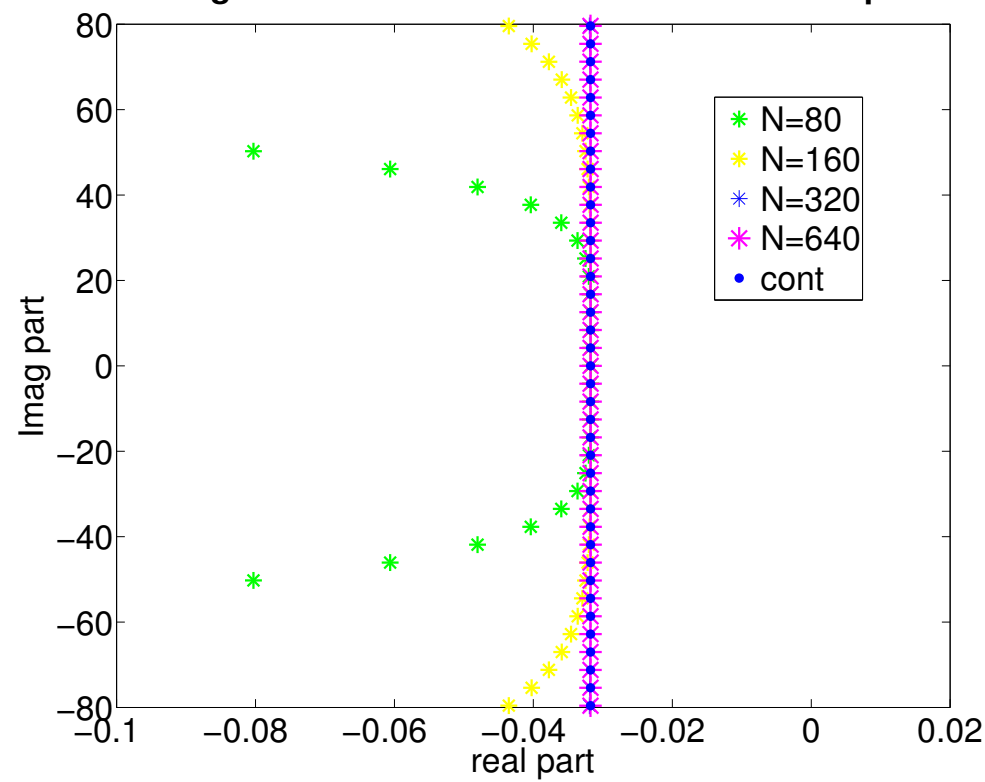

(b)

Figure 5.3: Figure 5.3a is a close-up of Figure 5.2. Figure 5.3b shows the same problem with added artificial dissipation. 


\section{Mimetic SBP properties}

Numerical instabilities in simulations of nonlinear problems have been studied for a long time. The false transfer of energy between different scales and the non-physical growth of numerical solutions was traced back to an inappropriate treatment of the nonlinear terms in [14]. In order to overcome this problem, Arakawa [1] proposed the use of a second order accurate mimetic finite-difference scheme. The principle behind his technique is the preservation of fundamental integral properties by using a combination of different discrete derivatives.

In the so called splitting technique [10,4], combinations of differential terms are used to correctly replicate the integration-by-parts and chain rule for products of convective terms. With this method, the Arakawa's technique can be reformulated using SBP operators to obtain stable high order schemes with mimetic properties.

\subsection{The vorticity equation}

Consider an incompressible two-dimensional inviscid fluid. The zero divergence condition implies the existence of a stream function $\psi$ related to the vorticity $\zeta$ by

$$
\zeta=\Delta \psi
$$

In this setting, the time evolution of the vorticity can be written as

$$
\frac{\partial \zeta}{\partial t}+J(\psi, \zeta)=0
$$

The function $J(\cdot, \cdot)$ in $(6.2)$ is the Jacobian differential operator given by

$$
J(a, b)=\frac{\partial a}{\partial x} \frac{\partial b}{\partial y}-\frac{\partial a}{\partial y} \frac{\partial b}{\partial x} .
$$

It is a skew-symmetric operator since $J(a, b)=-J(b, a)$. Moreover, for periodic functions $a$ and $b$ on the domain $\Omega$

$$
\int_{\Omega} J(a, b) d x d y=\int_{\Omega} a J(a, b) d x d y=\int_{\Omega} b J(a, b) d x d y=0 .
$$

These properties follow directly from integration-by-parts and periodic boundary conditions. 


\subsubsection{Conservation properties}

The mean kinetic energy for equation (6.2) is given by

$$
K=\frac{1}{2} \overline{(\nabla \psi)^{2}}
$$

where $\bar{f}=\int_{D} f d x d y /|D|$. The enstrophy (mean square vorticity) for equation (6.2) is defined as

$$
G=\frac{1}{2} \overline{\zeta^{2}}
$$

The vorticity equation (6.2) conserves the mean kinetic energy (6.5) and the enstrophy (6.6). The conservation of these quantities stems from the integral property (6.4), which, ultimately, comes from the integration-by-parts rule.

\subsubsection{Operator splitting}

An alternative expression for the Jacobian can be constructed as a linear combination of the following equivalent operators

$$
\begin{aligned}
& J_{1}(\psi, \zeta)=\left(\frac{\partial \psi}{\partial x} \frac{\partial \zeta}{\partial y}-\frac{\partial \psi}{\partial y} \frac{\partial \zeta}{\partial x}\right), \\
& J_{2}(\psi, \zeta)=\left(\frac{\partial}{\partial x}\left(\psi \frac{\partial \zeta}{\partial y}\right)-\frac{\partial}{\partial y}\left(\psi \frac{\partial \zeta}{\partial x}\right)\right), \\
& J_{3}(\psi, \zeta)=\left(\frac{\partial}{\partial y}\left(\zeta \frac{\partial \psi}{\partial x}\right)-\frac{\partial}{\partial x}\left(\zeta \frac{\partial \psi}{\partial y}\right)\right) .
\end{aligned}
$$

The general linear combination

$$
J(\psi, \zeta)=\alpha J_{1}+\beta J_{2}+\gamma J_{3}
$$

defines a new Jacobian operator equivalent to (6.3) for any set of coefficients $\alpha, \beta$ and $\gamma$ satisfying $\alpha+\beta+\gamma=1$.

\subsection{Jacobian operators on SBP form}

In order to define the discrete SBP version of the nonlinear operator $J(\psi, \zeta)$ in (6.8), we need new notation. For any vector $\mathbf{a}=\left(a_{1}, \ldots, a_{N}\right)$, let $\operatorname{diag}(\mathbf{a})$ be a diagonal matrix with the components of $\mathbf{a}$ as diagonal elements and $\mathbf{1}=$ $(1, \cdots, 1)^{T}$ be a vector of the same length as $\mathbf{a}$.

The discrete versions of (6.7) using SBP operators are given by

$$
\begin{aligned}
\mathbf{J}_{1}(\boldsymbol{\psi}, \boldsymbol{\zeta}) & =\left\{\left[\operatorname{diag}\left(P_{x}^{-1} Q_{x} \otimes I_{y} \boldsymbol{\psi}\right) \operatorname{diag}\left(I_{x} \otimes P_{y}^{-1} Q_{y} \boldsymbol{\zeta}\right)\right] \mathbf{1}\right. \\
& \left.-\left[\operatorname{diag}\left(I_{x} \otimes P_{y}^{-1} Q_{y} \boldsymbol{\psi}\right) \operatorname{diag}\left(P_{x}^{-1} Q_{x} \otimes I_{y} \boldsymbol{\zeta}\right)\right] \mathbf{1}\right\}
\end{aligned}
$$




$$
\begin{aligned}
\mathbf{J}_{2}(\boldsymbol{\psi}, \boldsymbol{\zeta}) & =\left\{\left(P_{x}^{-1} Q_{x} \otimes I_{y}\right)\left[\operatorname{diag}(\boldsymbol{\psi}) \operatorname{diag}\left(I_{x} \otimes P_{y}^{-1} Q_{y} \boldsymbol{\zeta}\right)\right] \mathbf{1}\right. \\
- & \left.\left(I_{x} \otimes P_{y}^{-1} Q_{y}\right)\left[\operatorname{diag}(\boldsymbol{\psi}) \operatorname{diag}\left(P_{x}^{-1} Q_{x} \otimes I_{y} \boldsymbol{\zeta}\right)\right] \mathbf{1}\right\}, \\
\mathbf{J}_{3}(\boldsymbol{\psi}, \boldsymbol{\zeta})= & \left\{\left(I_{x} \otimes P_{y}^{-1} Q_{y}\right)\left[\operatorname{diag}(\boldsymbol{\zeta}) \operatorname{diag}\left(P_{x}^{-1} Q_{x} \otimes I_{y} \boldsymbol{\psi}\right)\right] \mathbf{1}\right. \\
- & \left.\left(P_{x}^{-1} Q_{x} \otimes I_{y}\right)\left[\operatorname{diag}(\boldsymbol{\zeta}) \operatorname{diag}\left(I_{x} \otimes P_{y}^{-1} Q_{y} \boldsymbol{\psi}\right)\right] \mathbf{1}\right\},
\end{aligned}
$$

where $\boldsymbol{\zeta}$ and $\boldsymbol{\psi}$ are vectors with the same size of the mesh. In (6.9)-(6.11), the matrices $Q_{x, y}$ are skew-symmetric operators satisfying $Q_{x, y}+Q_{x, y}^{T}=0$.

Thus, the discretisation of (6.8) is obtained by

$$
\mathbf{J}=\alpha \mathbf{J}_{1}+\beta \mathbf{J}_{2}+\gamma \mathbf{J}_{3},
$$

where, for consistency, $\alpha+\beta+\gamma=1$.

In Paper II, it is proved that (6.12) is skew-symmetric and conserves discrete enstrophy and kinetic energy if and only if

$$
\alpha=\beta=\gamma=\frac{1}{3} .
$$

This choice gives the discrete mimetic SBP Jacobian of arbitrary order of accuracy as

$$
\mathbf{J}^{*}=\frac{1}{3}\left[\mathbf{J}_{1}+\mathbf{J}_{2}+\mathbf{J}_{3}\right] .
$$

It is also shown that the second order accurate version of (6.13) is the same as the Jacobian derived in [1].

\subsubsection{The order of accuracy}

The new Jacobian in (6.13) was tested for accuracy using the manufactured solution [15]

$$
\psi(x, y, t)=K[\sin (2 \pi(a x-t)+\cos (2 \pi(b y-t))] .
$$

The forced equation $\zeta_{t}+J(\psi, \zeta)=F$, was obtained by inserting (6.14) into (6.2).

Skew-symmetric SBP operators with accuracy order 2, 4, 6 and 8 were used in space and the standard fourth order Runge-Kutta in time. In all computations, we used $a=2$ and $b=3$. The results are presented in Table 6.1 (from Paper II) and show that all schemes converge with the design order of accuracy. Note that, the schemes converge with the interior order of accuracy since no boundary closure exists in this periodic case. 


\begin{tabular}{l|ll|ll|ll|lll}
\hline & SBP 2nd & & SBP 4th & & SBP 6th & & SBP 8th & \\
$\mathrm{N}$ & Err & $p$ & Err & $p$ & Err & $p$ & Err & $p$ \\
\hline 30 & $2.110^{-2}$ & - & $6.610^{-4}$ & - & $1.010^{-4}$ & - & $8.110^{-6}$ & - \\
40 & $1.110^{-2}$ & 1.97 & $4.310^{-4}$ & 3.95 & $1.810^{-5}$ & 5.88 & $8.510^{-7}$ & 7.83 \\
50 & $7.510^{-3}$ & 1.94 & $1.710^{-4}$ & 3.94 & $4.910^{-6}$ & 5.93 & $1.410^{-7}$ & 7.90 \\
60 & $5.310^{-3}$ & 2.03 & $8.610^{-5}$ & 3.99 & $1.610^{-6}$ & 5.95 & $3.410^{-8}$ & 7.93 \\
70 & $3.910^{-3}$ & 2.00 & $4.610^{-5}$ & 3.98 & $6.510^{-7}$ & 5.96 & $1.010^{-8}$ & 7.95 \\
80 & $1.910^{-3}$ & 1.97 & $2.710^{-5}$ & 3.98 & $5.910^{-7}$ & 5.97 & $3.410^{-9}$ & 7.96 \\
\hline
\end{tabular}

Table 6.1: Discrete $L^{2}$-error and rate of convergence for the 2 nd, 4 th, 6 th and 8 th SBP operators. The error is indicated with $E r r$ and the order of accuracy with $p$. $\mathrm{N}$ indicates the number of grid points in the $x$ and $y$ direction, respectively. 


\section{Boundary conditions for the Navier-Stokes equations}

The incompressible Navier-Stokes equations can be considered in various formulations. Although they are mathematically equivalent in the continuous setting, each formulation can have different advantages or drawbacks in the discrete case. For instance, the velocity-pressure formulation requires special artificial techniques to preserve zero divergence. In the velocity-divergence formulation, the divergence relation provides automatically a divergence-free solution to the order of the scheme, and this formulation will be analysed below.

\subsection{The continuous problem}

Consider the two-dimensional incompressible Navier-Stokes equations

$$
\tilde{I} \mathbf{v}_{t}+A \mathbf{v}_{x}+B \mathbf{v}_{y}-\epsilon \tilde{I}\left(\mathbf{v}_{x x}+\mathbf{v}_{y y}\right)=0, \quad(x, y) \in \Omega, t \geq 0,
$$

where $\mathbf{v}=(u, v, p)^{T}$ denotes the velocity field $(u, v)$ and pressure $p$. In (7.1), $\epsilon$ indicates the viscosity, $\Omega$ is a bounded domain and the coefficient matrices are

$$
A(u)=\left[\begin{array}{lll}
u & 0 & 1 \\
0 & u & 0 \\
1 & 0 & 0
\end{array}\right], B(v)=\left[\begin{array}{lll}
v & 0 & 0 \\
0 & v & 1 \\
0 & 1 & 0
\end{array}\right] \text { and } \tilde{I}=\left[\begin{array}{ccc}
1 & 0 & 0 \\
0 & 1 & 0 \\
0 & 0 & 0
\end{array}\right]
$$

To be able to apply the energy method, the nonlinear convection terms in (7.1) must be split as

$$
A \mathbf{v}_{x}=\frac{1}{2}(A \mathbf{v})_{x}+\frac{1}{2} A \mathbf{v}_{x}-\frac{1}{2} A_{x} \mathbf{v}, \quad B \mathbf{v}_{y}=\frac{1}{2}(B \mathbf{v})_{y}+\frac{1}{2} B \mathbf{v}_{y}-\frac{1}{2} B_{y} \mathbf{v} .
$$

By inserting (7.3) into (7.1) and using the divergence relation, the incompressible Navier-Stokes equations in skew-symmetric form are given by

$$
\tilde{I} \mathbf{v}_{t}+\frac{1}{2}\left[(A \mathbf{v})_{x}+A \mathbf{v}_{x}+(B \mathbf{v})_{y}+B \mathbf{v}_{y}\right]-\epsilon \tilde{I} \Delta \mathbf{v}=0,(x, y) \in \Omega, t>0 .
$$

The IBVP for (7.4) is obtained by adding initial and boundary conditions of the form

$$
\mathbf{v}(x, y, 0)=f(x, y), \quad(x, y) \in \Omega
$$


and

$$
H \mathbf{v}(x, y, t)=g(t), \quad(x, y) \in \partial \Omega, t \geq 0 .
$$

In (7.5)-(7.6), $f$ and $g$ are smooth compatible initial and boundary data, respectively. The boundary operator $H$ in (7.6) is in general a rectangular matrix including differential operators. It can represent Dirichlet, Neumann or Robin type of boundary conditions.

In this chapter, we are interested in boundary conditions of the special form

$$
H \mathbf{v}=W^{-}-R W^{+}=\mathbf{g},
$$

where $R$ is a rectangular matrix. This formulation introduces generalized ingoing variables, $W^{-}$, specified in terms of generalized outgoing variables, $W^{+}$, and given data $\mathbf{g}$.

\subsubsection{The energy method and the boundary conditions}

The energy method applied to (7.4) together with Green's formula yields

$$
\frac{d}{d t}\|\mathbf{v}\|_{\tilde{I}}^{2}+2 \epsilon\|\nabla \mathbf{v}\|_{\tilde{I}}^{2}=\mathrm{BT},
$$

where $\|\mathbf{v}\|_{\tilde{I}}^{2}=\int_{\Omega} \mathbf{v}^{T} \tilde{I} \mathbf{v}$ is a semi-norm which allows for $\|\mathbf{v}\|_{\tilde{I}}=0$ even for $p \neq 0$. In (7.8), BT denotes the boundary term given by

$$
\mathrm{BT}=-\oint_{\Omega} \mathbf{w}^{T} A_{\mathbf{n}} \mathbf{w} d s
$$

where

$$
\mathbf{w}=\left[\begin{array}{c}
u_{n} \\
u_{s} \\
p \\
\epsilon \partial_{\mathbf{n}} u_{n} \\
\epsilon \partial_{\mathbf{n}} u_{s}
\end{array}\right], \quad A_{\mathbf{n}}=\left[\begin{array}{ccccc}
u_{n} & 0 & 1 & -1 & 0 \\
0 & u_{n} & 0 & 0 & -1 \\
1 & 0 & 0 & 0 & 0 \\
-1 & 0 & 0 & 0 & 0 \\
0 & -1 & 0 & 0 & 0
\end{array}\right]
$$

and $d s=\sqrt{d x^{2}+d y^{2}}$. In (7.10), $u_{n}$ and $u_{s}$ denote the outward normal and tangential velocity components, respectively,

To derive an energy estimate for (7.8), we must bound BT with a minimal set of boundary conditions. By diagonalising BT, (7.9) can be rewritten as

$$
\mathrm{BT}=-\oint\left[\begin{array}{l}
W^{+} \\
W^{-}
\end{array}\right]^{T}\left[\begin{array}{cc}
\Lambda^{+} & 0 \\
0 & \Lambda^{-}
\end{array}\right]\left[\begin{array}{l}
W^{+} \\
W^{-}
\end{array}\right]
$$

where $\Lambda^{-}$and $\Lambda^{+}$contain the negative and positive diagonal elements, and $W^{-}$ and $W^{+}$indicate the corresponding variables. The minimal number of boundary conditions is equal to the number of negative diagonal entries. 
In Paper III, the matrix $A_{\mathbf{n}}$ in (7.10) was diagonalised using two different techniques: eigenvalue decomposition and non-singular rotations. Both techniques lead to matrices $\Lambda^{-}$with two negative diagonal entries. This implies that two boundary conditions are needed.

The number of boundary conditions is independent of the specific transformation used to arrive at the diagonal form (7.11), as long as the resulting variables are linearly independent. This follows from Sylvester's law of inertia.

The two transformations mentioned above lead to different forms of $W^{-}$and $W^{+}$in (7.7). In the first case, the positive and negative eigenvectors define the generalised in- and outgoing characteristic variables

$$
W^{-}=\left[\begin{array}{c}
\lambda_{1} u_{n}+p-\epsilon \partial_{\mathbf{n}} u_{n} \\
\lambda_{2} u_{s}-\epsilon \partial_{\mathbf{n}} u_{s}
\end{array}\right], \quad W^{+}=\left[\begin{array}{c}
\lambda_{4} u_{s}-\epsilon \partial_{\mathbf{n}} u_{s} \\
\lambda_{5} u_{n}+p-\epsilon \partial_{\mathbf{n}} u_{n}
\end{array}\right]
$$

In the second case, at an inflow boundary $\left(u_{n}<0\right)$ we have

$$
W^{-}=\left[\begin{array}{c}
u_{n}^{2}+p-\epsilon \partial_{\mathbf{n}} u_{n} \\
u_{n} u_{s}-\epsilon \partial_{\mathbf{n}} u_{s}
\end{array}\right], \quad W^{+}=\left[\begin{array}{c}
p-\epsilon \partial_{\mathbf{n}} u_{n} \\
\epsilon \partial_{\mathbf{n}} u_{s}
\end{array}\right] .
$$

At an outflow boundary $\left(u_{n} \geq 0\right)$, they are the same, but interchanged. In both situations, $W^{-}$and $W^{+}$are called the generalised in- and outgoing rotated variables.

Finally, the general form (7.7) is obtained by decomposing $H$ as

$$
H \mathbf{v}=\left(H^{+}-R H^{-}\right) \mathbf{v}=W^{-}-R W^{+} .
$$

Here, $\mathrm{H}^{-}$and $\mathrm{H}^{+}$are suitable boundary operators which transform the original vector variable $\mathbf{v}$ into the generalised in- and outgoing variables in one or the other formulation. The two formulations provide completely general sets of combinations of variables and their derivatives. They can be adapted to yield far field, solid wall and "no pressure" type of conditions. The so called "no pressure" boundary conditions do not include the pressure.

Note that, in both formulations, the boundary conditions are nonlinear. Note also that, in the rotated formulation, the variables change at an inflow or outflow boundary. This means that the boundary procedure varies in time.

The boundary conditions (7.12), in both the characteristic and rotated formulation, lead to the following two results.

Proposition 7.1.1. The boundary conditions (7.12) bounds (7.11) if

$$
\Lambda^{+}+R^{T} \Lambda^{-} R>0
$$

and a positive semi-definite or positive definite matrix $\Gamma$ exists such that

$$
-\Lambda^{-}+\left(\Lambda^{-} R\right)\left[\Lambda^{+}+R^{T} \Lambda^{-} R\right]^{-1}\left(\Lambda^{-} R\right)^{T} \leq \Gamma<\infty .
$$


Corollary 7.1.1. The homogeneous boundary conditions $W^{-}=R W^{+}$lead to a bound for (7.11) if

$$
\Lambda^{+}+R^{T} \Lambda^{-} R \geq 0 .
$$

In [11], similar results were proved for linear IBVPs, while, in Paper III, they were extended to the nonlinear incompressible Navier-Stokes problem.

\subsection{The discrete problem}

In this section, stable semi-discrete and fully discrete SBP-SAT approximations of (7.4) with weakly imposed boundary and initial conditions are presented. The derivation of the discrete problem follows the continuous analysis in a stepby-step manner.

\subsubsection{The semi-discrete formulation}

Consider a square domain and indicate the west, east, south and north boundaries by $b \in\{\mathrm{W}, \mathrm{E}, \mathrm{S}, \mathrm{N}\}$ and the corresponding normals by $\mathbf{n}^{b}=\left(n_{x}^{b}, n_{y}^{b}\right)$. We also introduce the time-dependent vector variable $\mathbf{V}=(\mathbf{u}(t), \mathbf{v}(t), \mathbf{p}(t))^{T}$ and the discrete version of $(7.2)$

$$
\mathbf{A}=\left[\begin{array}{ccc}
\mathrm{D}(\mathbf{u}) & \mathbf{0} & I_{N M} \\
\mathbf{0} & \mathrm{D}(\mathbf{u}) & \mathbf{0} \\
I_{N M} & \mathbf{0} & \mathbf{0}
\end{array}\right], \mathbf{B}=\left[\begin{array}{ccc}
\mathrm{D}(\mathbf{v}) & \mathbf{0} & \mathbf{0} \\
\mathbf{0} & \mathrm{D}(\mathbf{v}) & I_{N M} \\
\mathbf{0} & I_{N M} & \mathbf{0}
\end{array}\right], \tilde{I}_{3}=\left[\begin{array}{ccc}
I_{N M} & \mathbf{0} & \mathbf{0} \\
\mathbf{0} & I_{N M} & \mathbf{0} \\
\mathbf{0} & \mathbf{0} & \mathbf{0}
\end{array}\right]
$$

Here, $\mathbf{A}, \mathbf{B}$ and $\tilde{I}_{3}$ are $3 N M \times 3 N M$ matrices while $\mathbf{0}$ is a $N M \times N M$ matrix of zeros. The operator $D(\mathbf{u})$ is a diagonal matrix with the components of $\mathbf{u}$ as diagonal elements.

With this notation, the semi-discrete SBP-SAT approximation of (7.4) with weakly imposed boundary conditions can be written

$$
\tilde{I}_{3} \mathbf{V}_{t}+\tilde{D}_{x} \mathbf{V}+\tilde{D}_{y} \mathbf{V}-\epsilon\left[\left(\tilde{I} \otimes D_{x}\right)^{2}+\left(\tilde{I} \otimes D_{y}\right)^{2}\right] \mathbf{V}=\operatorname{Pen}_{\mathrm{BT}},
$$

where the skew-symmetric splitting (7.3) is approximated by the difference operators

$$
\tilde{D}_{x}=\frac{1}{2}\left(I_{3} \otimes D_{x}\right) \mathbf{A}+\frac{1}{2} \mathbf{A}\left(I_{3} \otimes D_{x}\right), \quad \tilde{D}_{y}=\frac{1}{2}\left(I_{3} \otimes D_{y}\right) \mathbf{B}+\frac{1}{2} \mathbf{B}\left(I_{3} \otimes D_{y}\right) .
$$

In (7.13), Pen ${ }_{\mathrm{BT}}$ is the penalty term that imposes the boundary conditions. It can be decomposed into the sum of four terms corresponding to each boundary of the square domain, namely $\operatorname{Pen}_{\mathrm{BT}}=\operatorname{Pen}_{\mathrm{BT}}^{W}+\operatorname{Pen}_{\mathrm{BT}}^{E}+\operatorname{Pen}_{\mathrm{BT}}^{S}+\operatorname{Pen}_{\mathrm{BT}}^{N}$, where

$$
\begin{aligned}
& \operatorname{Pen}_{\mathrm{BT}}^{W}=\left(I_{3} \otimes P_{x}^{-1} E_{0} \otimes I_{M}\right) \boldsymbol{\Sigma}^{W}\left(\mathbf{H}^{W} \mathbf{V}-G^{W}\right), \\
& \operatorname{Pen}_{\mathrm{BT}}^{E}=\left(I_{3} \otimes P_{x}^{-1} E_{N} \otimes I_{M}\right) \boldsymbol{\Sigma}^{E}\left(\mathbf{H}^{E} \mathbf{V}-G^{E}\right), \\
& \operatorname{Pen}_{\mathrm{BT}}^{S}=\left(I_{3} \otimes I_{N} \otimes P_{y}^{-1} E_{0}\right) \Sigma^{S}\left(\mathbf{H}^{S} \mathbf{V}-G^{S}\right), \\
& \operatorname{Pen}_{\mathrm{BT}}^{N}=\left(I_{3} \otimes I_{N} \otimes P_{y}^{-1} E_{M}\right) \boldsymbol{\Sigma}^{N}\left(\mathbf{H}^{N} \mathbf{V}-G^{N}\right) .
\end{aligned}
$$


Here, $\mathbf{H}^{W, E, S, N}$ are discrete versions of $H$ in (7.12) and they can formally be written as

$$
\mathbf{H}^{b} \mathbf{V}=\mathbf{H}^{-, b} \mathbf{V}-\mathbf{R} \mathbf{H}^{+, b} \mathbf{V}=\mathbf{W}^{-, b}-\mathbf{R} \mathbf{W}^{+, b} .
$$

The vectors $G^{W, E, S, N}$ contain the boundary data at the appropriate boundary points and $\boldsymbol{\Sigma}^{W, E, S, N}$ are penalty matrices.

The following two results were proven in Paper III.

Proposition 7.2.1. The semi-discrete approximation (7.13) of (7.4) with penalty matrices

$$
\boldsymbol{\Sigma}^{b}=\left(\boldsymbol{H}^{-, b}\right)^{T} \boldsymbol{\Lambda}^{-, b},
$$

and boundary operators ( 7.15$)$ leads to stability if

$$
\Lambda^{+, b}+\boldsymbol{R}^{T} \boldsymbol{\Lambda}^{-, b} \boldsymbol{R}>0
$$

holds

Corollary 7.2.1. The semi-discrete approximation (7.13) of (7.4) with penalty matrix (7.16) and boundary operators (7.15) with homogeneous boundary conditions leads to stability if

$$
\boldsymbol{\Lambda}^{+, b}+\boldsymbol{R}^{T} \boldsymbol{\Lambda}^{-, b} \boldsymbol{R} \geq 0
$$

\subsubsection{The fully-discrete formulation}

Consider the fully-discrete variable $\mathbf{V}=\left[\mathbf{V}_{1}, \ldots, \mathbf{V}_{k}, \ldots, \mathbf{V}_{L}\right]^{T}$, where each $\mathbf{V}_{k}=$ $\left(\mathbf{u}_{k}, \mathbf{v}_{k}, \mathbf{p}_{k}\right)^{T}$ represents the variables on the whole spatial domain at the $k$-th time level. The SBP-SAT approximation of (7.4) including a weak imposition of the boundary and initial conditions can be written

$$
\left(D_{t} \otimes \tilde{I}_{3}\right) \mathbf{V}+\mathbf{F}(\mathbf{V}) \mathbf{V}=\mathbf{P e n}_{\mathrm{BT}}+\mathbf{P e n}_{\text {Time }} .
$$

Here, $\mathbf{F}=\operatorname{blockdiag}\left(F\left(\mathbf{V}_{0}\right), \ldots, F\left(\mathbf{V}_{L}\right)\right)$ where the blocks are the nonlinear spatial differential operators given by

$$
F\left(\mathbf{V}_{k}\right)=\left(\tilde{D}_{x}\right)_{k}+\left(\tilde{D}_{y}\right)_{k}-\epsilon\left[\left(\tilde{I} \otimes D_{x}\right)^{2}+\left(\tilde{I} \otimes D_{y}\right)^{2}\right], \quad k=0, \ldots, L .
$$

In (7.17), $\mathbf{P e n}_{\mathrm{BT}}$ is a vector of penalty terms for imposing the boundary conditions weakly at each time level, i.e.

$$
\operatorname{Pen}_{\mathrm{BT}}=\left[\left(\mathrm{Pen}_{\mathrm{BT}}\right)_{0}, \ldots,\left(\mathrm{Pen}_{\mathrm{BT}}\right)_{k}, \ldots,\left(\mathrm{Pen}_{\mathrm{BT}}\right)_{L}\right]^{T} .
$$

Each $\left(\operatorname{Pen}_{\mathrm{BT}}\right)_{k}$ is the sum of the four boundary penalties given in (7.14), considering $\mathbf{V}_{k}$ as the discrete variable at the time level $k$. Finally, $\mathbf{P e n}_{\text {Time }}$ is the penalty term for imposing the initial condition weakly given by

$$
\mathbf{P e n}_{\text {Time }}=\sigma_{t}\left(P_{t}^{-1} E_{0} \otimes \tilde{I} \otimes I_{N} \otimes I_{M}\right)(\mathbf{V}-\mathbf{f}),
$$

where $\mathbf{f}$ is a vector of the same length as $\mathbf{V}$ containing the initial data at $k=0$. The following result is proven is Paper III. 
Proposition 7.2.2. The discretization (7.17) of (7.4), with spatial penalty terms (7.18) satisfying the assumptions of Proposition 7.2.1 (or Corollary 7.2.1) and the temporal penalty term (7.19) is stable if $\sigma_{t}=-1$.

\subsection{Spectral analysis}

In this section (and in Paper IV), we discuss the continuous and discrete spectrum of the linearised Navier-Stokes problem. In particular, we show how to use the spectrum in order to predict the convergence rate to steady state.

\subsubsection{The continuous and discrete spectrum}

Consider (7.1) linearised around a constant state $\overline{\mathbf{v}}=(\bar{u}, \bar{v}, \bar{p})^{T}$ on the strip $0 \leq x \leq 1,-\infty \leq y \leq \infty$. The continuous and discrete spectrum of the associated IBVP with homogeneous boundary conditions can be derived using the Fourier-Laplace technique [6]. Details on how to compute the spectrum are given in Paper IV.

Let $\hat{\mathrm{v}}=\hat{\mathrm{v}}(x, \omega, s)$ be the solution to the Fourier-Laplace transformed equations, where $i \omega, \omega \in \mathbb{R}$, is the dual variable to $y$ and $s \in \mathbb{C}$ is the dual variable to time. Let the eigenvalue of the continuous spectrum with the largest real part be $s^{*}=\eta^{*}+i \xi^{*}$. By applying the inverse Laplace transform to $\hat{\mathrm{v}}$, we get

$$
\tilde{\mathrm{v}}(x, \omega, t)=\exp \left(\eta^{*} t\right)\left(\frac{1}{2 \pi} \int_{-\infty}^{+\infty} \hat{\mathrm{v}}\left(\eta^{*}+i \xi\right) \exp (i \xi) d \xi\right)
$$

If there are no eigenvalues with non-negative real part, i.e. $\eta^{*}<0$, the convergence to steady state of the solution follows directly from (7.20) and Parseval's relation. From now on, we denote $\eta^{*}$ as the continuous decay rate.

In concert with the continuous case, the discrete decay rate is $\eta_{d}^{*}=\operatorname{Re}\left(s_{d}^{*}\right)$, where $s_{d}^{*}$ is the discrete eigenvalue with the largest real part.

Figure 7.1 (from Paper IV) shows a comparison between the continuous and discrete spectra for $\bar{u}=1, \bar{v}=0, \epsilon=0.01$ and $\omega=10$. The discrete spectra are computed using $N=30,50,70$ grid points and SBP42 operators. As can be seen, the spectra consist of eigenvalues with negative real parts, thus, the decay rates are negative. Hence, the convergence to steady state is guaranteed. Furthermore, it can be seen that the discrete spectrum converges to the continuous one as the mesh size decreases. In particular, this is true for the most right located eigenvalues, i.e., the discrete decay rate converges to the continuous one as the mesh size decreases. Similar results were obtained with other higher order accurate SBP operators. 


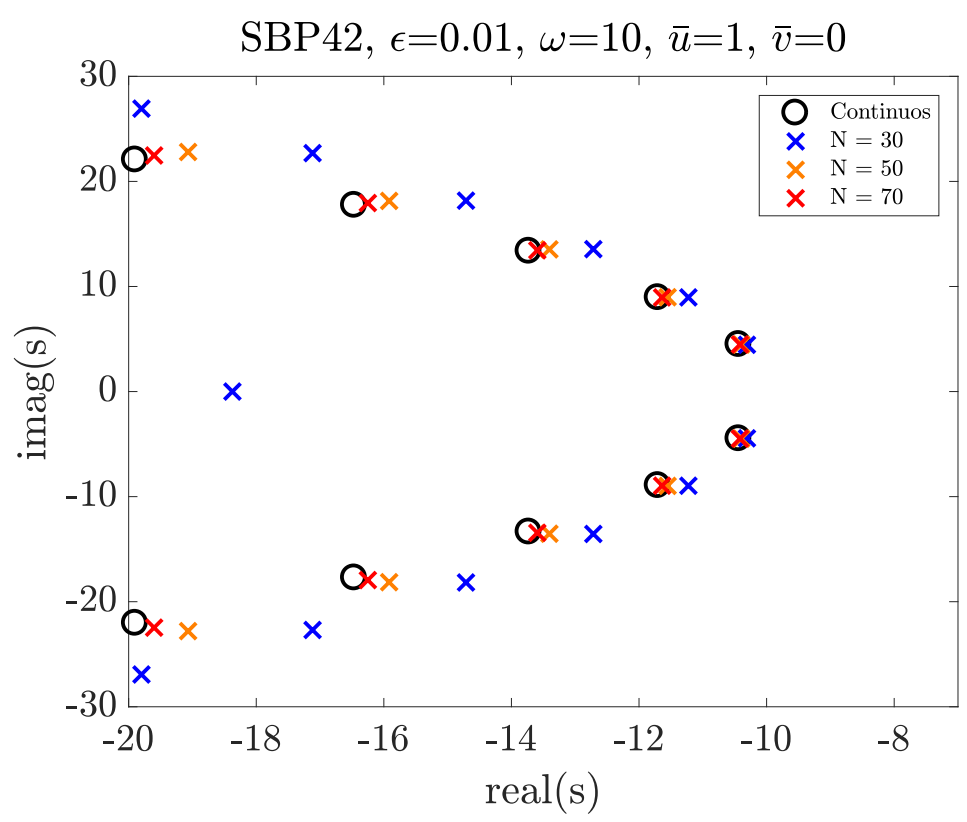

Figure 7.1: Comparison between the continuous and discrete spectra.

\subsubsection{Predicted and practical convergence rates}

The decay rates discussed above can be used to predict the convergence rate to steady state. To exemplify, we consider numerical results obtained from the linearised IBVP (7.1) equipped with homogeneous boundary condition and the initial condition $u(x, y)=v(x, y)=x^{2}(x-1)^{2} \sin (2 \pi \omega y)$.

In Figures 7.2a-7.2b (from paper IV), we compare different SBP-SAT approximations of the problem at fixed meshes with $N=30,70$ grid points. The considered operators are SBP21, SBP42, and SBP84 with accuracy of order 2, 3 and 5 , respectively. The theoretical decay rate (represented by the black line in the figures) is obtained using the frequency $\omega=10$ is $e^{\eta_{10}^{*} t}$ with $\eta_{10}^{*}=-10.4350$. The discrete theoretical decay rate (black line with stars) includes the dissipative effect of the time discretisation. The plot shows that the decay rates of the numerical steady state calculations approach the theoretical predictions as the mesh size decreases. Moreover, the high order ones were more accurate on fine grids.

\subsection{Comparison of different boundary conditions}

Since the discrete decay rate converges to the continuous one, we can compare different choices of boundary conditions by directly studying the continuous decay rate. 


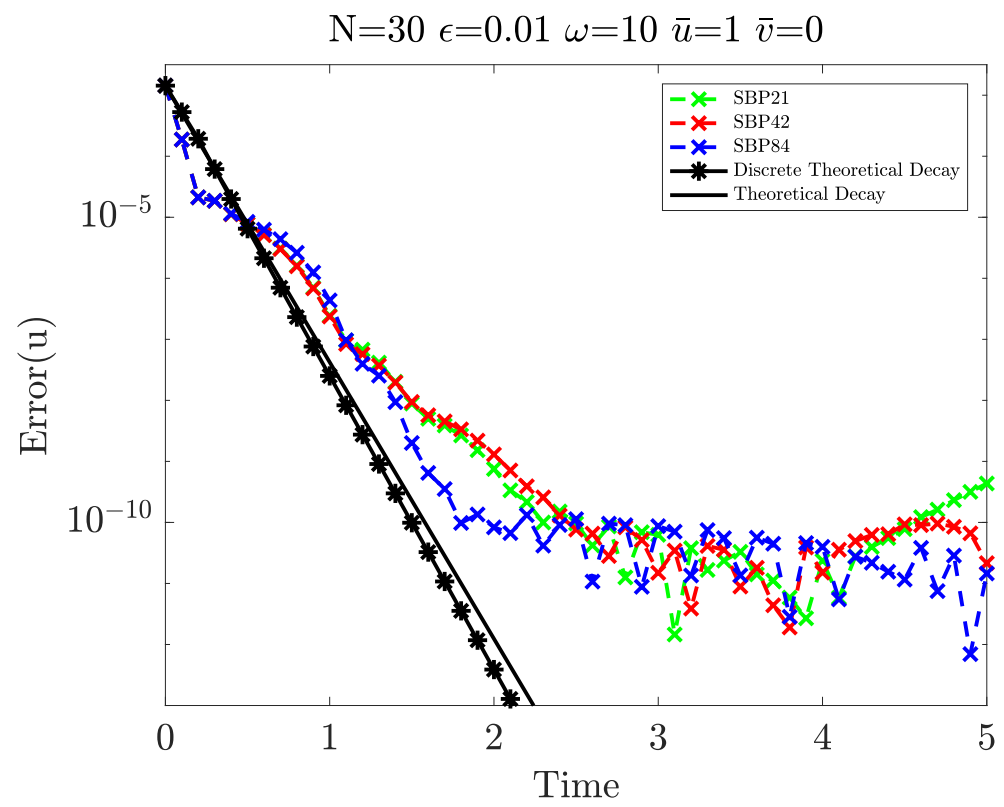

(a)

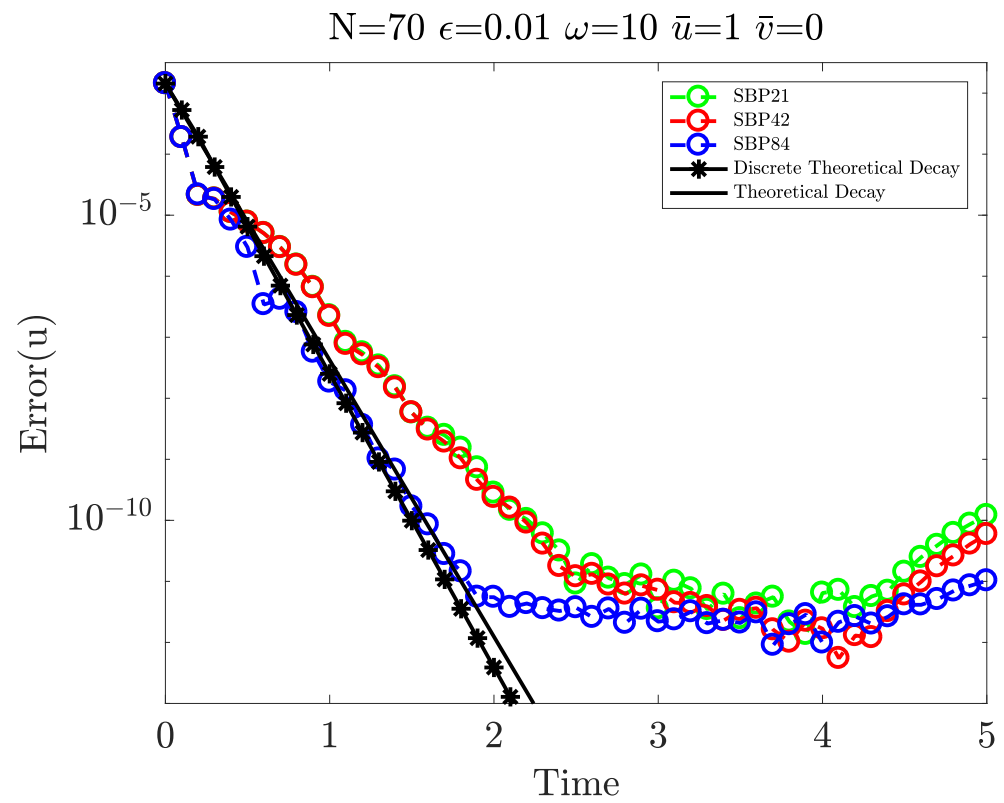

(b)

Figure 7.2: Practical and theoretical decay rates. A comparison of different orders of accuracy for fixed meshes. 
We consider three types of boundary conditions, namely, purely in-going conditions $(R=0$ in $(7.7))$, solid wall and conditions (7.7) with $R$ such that no pressure data are imposed. We refer to the formulation with characteristic variables as $\mathrm{CBC}$, while we use $\mathrm{RBC}$ for the one with rotated variables.

In Figures 7.3a-7.3b (from Paper IV), the decay rates obtained with both formulations are shown for $\epsilon=0.1$. It was found (somewhat surprisingly) that the least dissipative types of boundary conditions are the ones where only the ingoing variables are specified. Further investigations with smaller viscosity also revealed that, for this type of conditions, the $\mathrm{CBC}$ formulation converges faster to steady state. In the other cases with a non-zero $R$, the RBC formulation is preferable since the associated decay rates are significantly larger than in the CBC case. 


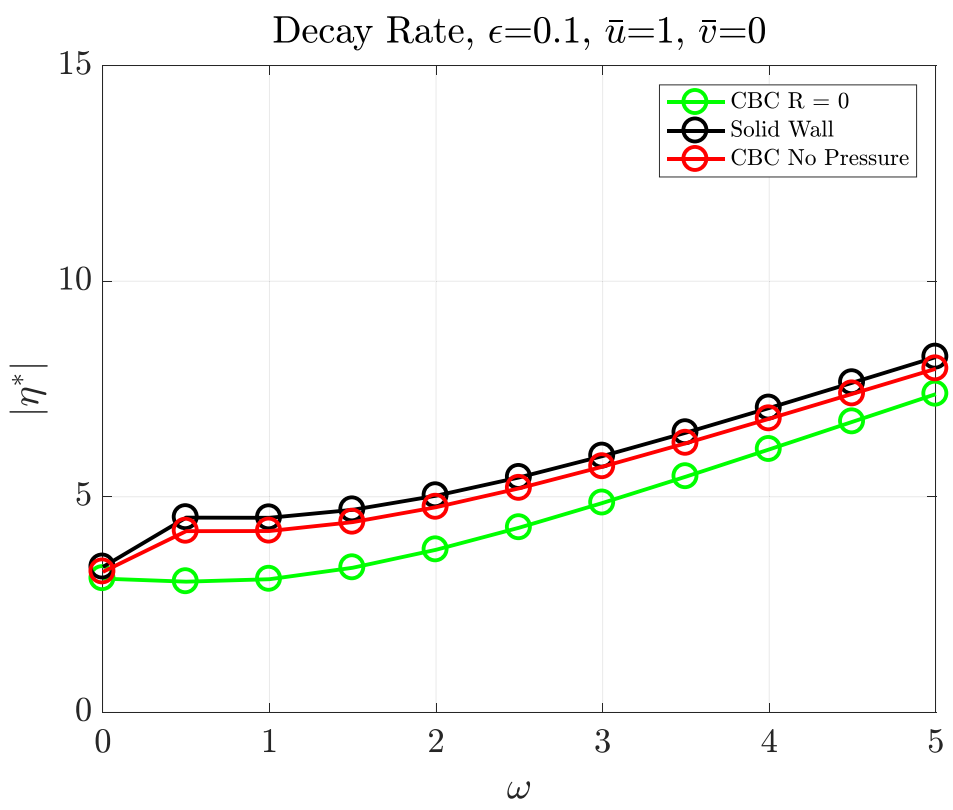

(a)

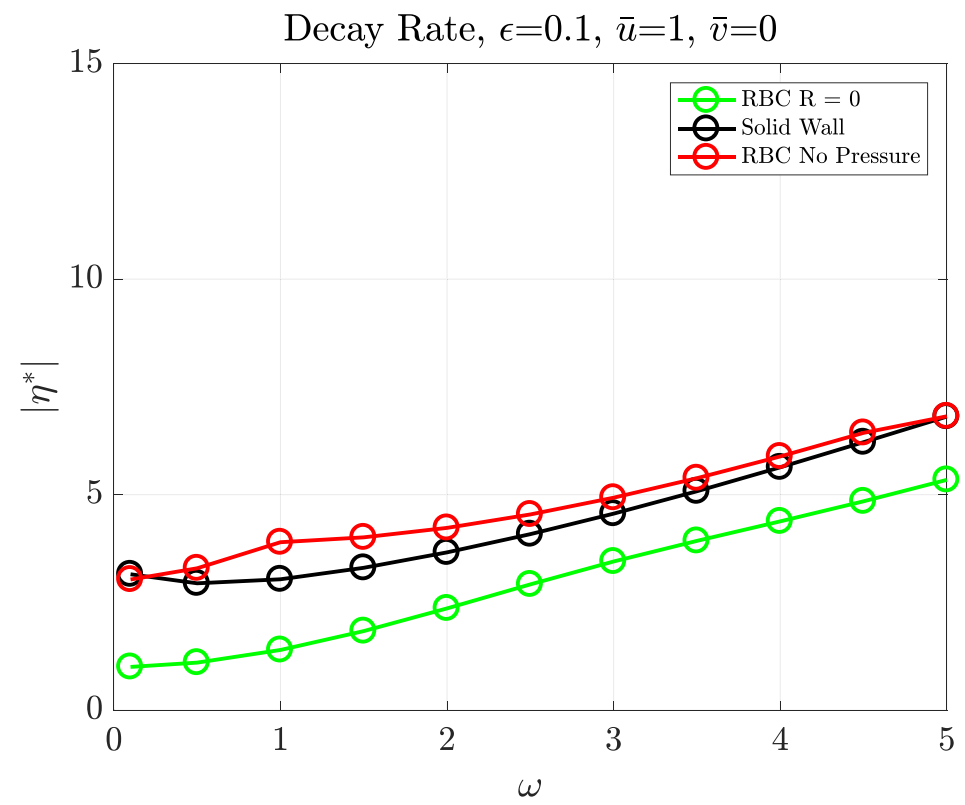

(b)

Figure 7.3: Comparison of different boundary conditions using characteristic variables (Figure 7.3a) and rotated variables (Figure 7.3b). 


\section{Summary of papers}

\section{Paper I: Well-posedness, stability and conserva- tion for a discontinuous interface problem}

Paper I deals with discontinuous linear advection problems where the solution propagates with different wave speeds in different regions satisfying jump conditions. An artificial interface is introduced which separates those regions and interface conditions are derived to guarantee stability and, optionally, conservation properties. A spectral analysis shows that by adding a suitable artificial dissipation operator to the SBP-SAT approximation with an interface treatment, the resulting discretisation is strictly stable.

\section{Paper II: A new high order energy and enstro- phy conserving Arakawa-like Jacobian differential operator}

In Paper II, the vorticity equation is considered. We prove by mimicking the integration-by-part rule in the discrete setting, that several properties (such as conservation of quantities) follow naturally. SBP operators are used to derive a new Arakawa-like Jacobian differential operator with mimetic properties by combining different consistent approximations of the convection terms. Skewsymmetry and kinetic energy and enstrophy conservation are obtained for periodic problems using schemes with arbitrarily high order of accuracy.

\section{Paper III: Energy stable boundary conditions for the nonlinear incompressible Navier-Stokes equa- tions}

Paper III is dedicated to the derivation of completely general and flexible energy stable boundary conditions for the nonlinear incompressible Navier-Stokes problem. Two different formulations are studied. A theoretical and numerical analysis are performed in order to provide continuous and discrete energy estimates and show that the boundary conditions can be adapted to different solid wall and far field situations. 


\section{Paper IV: Spectral analysis of the incompressible Navier-Stokes equations with different boundary conditions}

Paper IV extends the analysis in Paper III by providing a comparison of different boundary condition and investigating their effect on the spectral properties of the associated linearised incompressible Navier-Stokes problem. The theoretical and practical convergence to steady state are compared for different boundary conditions and formulations. 


\section{References}

[1] Akio Arakawa. Computational design for long-term numerical integration of the equations of fluid motion: Two-dimensional incompressible flow. part I. Journal of Computational Physics, 1(1):119-143, 1966.

[2] Douglas N Arnold, Franco Brezzi, Bernardo Cockburn, and L Donatella Marini. Unified analysis of discontinuous Galerkin methods for elliptic problems. SIAM journal on numerical analysis, 39(5):1749-1779, 2002.

[3] Mark H Carpenter, David Gottlieb, and Saul Abarbanel. Time-stable boundary conditions for finite-difference schemes solving hyperbolic systems: Methodology and application to high-order compact schemes. Journal of Computational Physics, 111(2):220-236, 1994.

[4] Travis C Fisher, Mark H Carpenter, Jan Nordström, Nail K Yamaleev, and Charles Swanson. Discretely conservative finite-difference formulations for nonlinear conservation laws in split form: Theory and boundary conditions. Journal of Computational Physics, 234:353-375, 2013.

[5] Bertil Gustafsson. On the implementation of boundary conditions for the method of lines. BIT Numerical Mathematics, 38(2):293-314, 1998.

[6] Heinz-Otto Kreiss and Jens Lorenz. Initial-boundary value problems and the Navier-Stokes equations, volume 47. Siam, 1989.

[7] Tomas Lundquist and Jan Nordström. The SBP-SAT technique for initial value problems. Journal of Computational Physics, 270:86-104, 2014.

[8] Ken Mattsson and Jan Nordström. Summation by parts operators for finite difference approximations of second derivatives. Journal of Computational Physics, 199(2):503-540, 2004.

[9] Ken Mattsson, Magnus Svärd, and Jan Nordström. Stable and accurate artificial dissipation. Journal of Scientific Computing, 21(1):57-79, 2004.

[10] Jan Nordström. Conservative finite difference formulations, variable coefficients, energy estimates and artificial dissipation. J Sci Comput., 29(3):375404, 2006.

[11] Jan Nordström. A Roadmap to Well Posed and Stable Problems in Computational Physics. J Sci Comput., 71(1):365-385, 2017.

[12] Jan Nordström and Tomas Lundquist. Summation-by-parts in time. Journal of Computational Physics, 251:487-499, 2013.

[13] Pelle Olsson. Summation by parts, projections, and stability. I. Mathematics of Computation, 64(211):1035-1065, 1995.

[14] Norman A Phillips. An example of non-linear computational instability. The Atmosphere and the Sea in motion, 501, 1959. 
[15] Lee Shunn, Frank Ham, and Parviz Moin. Verification of variable-density flow solvers using manufactured solutions. Journal of Computational Physics, 231(9):3801-3827, 2012.

[16] B. Strand. Summation by parts for finite difference approximations for d/dx. Journal of Computational Physics, 110:47-67, 1994.

[17] Magnus Svärd, Mark H Carpenter, and Jan Nordström. A stable high-order finite difference scheme for the compressible Navier-Stokes equations, farfield boundary conditions. Journal of Computational Physics, 225(1):10201038, 2007.

[18] Magnus Svärd and Jan Nordström. On the order of accuracy for difference approximations of initial-boundary value problems. Journal of Computational Physics, 218(1):333-352, 2006.

[19] Magnus Svärd and Jan Nordström. Review of summation-by-parts schemes for initial-boundary-value problems. Journal of Computational Physics, $268: 17-38,2014$. 


\section{Papers}

The papers associated with this thesis have been removed for copyright reasons. For more details about these see:

http://urn.kb.se/resolve?urn=urn:nbn:se:liu:diva-140497 